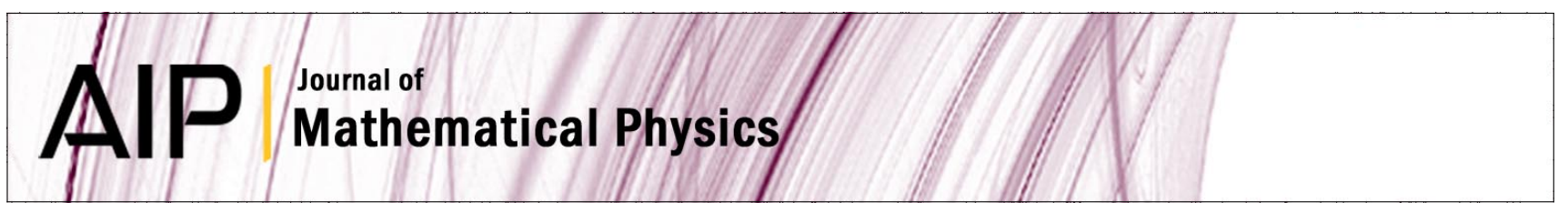

\title{
Global existence and nonrelativistic limit for the Vlasov-Maxwell-Chern- Simons system
}

Hyungjin Huh and Ho Lee

Citation: J. Math. Phys. 53, 103306 (2012); doi: 10.1063/1.4759129

View online: http://dx.doi.org/10.1063/1.4759129

View Table of Contents: http://jmp.aip.org/resource/1/JMAPAQ/v53/i10

Published by the American Institute of Physics.

\section{Related Articles}

Maxwell-Chern-Simons vortices on compact surfaces: Nonequivalence of the first and the second order equations

J. Math. Phys. 54, 023505 (2013)

Localization in abelian Chern-Simons theory

J. Math. Phys. 54, 023507 (2013)

The equivalence of the Chern-Simons-Schrödinger equations and its self-dual system

J. Math. Phys. 54, 021502 (2013)

Noncolliding Brownian motion with drift and time-dependent Stieltjes-Wigert determinantal point process

J. Math. Phys. 53, 103305 (2012)

Standing waves of the Schrödinger equation coupled with the Chern-Simons gauge field

J. Math. Phys. 53, 063702 (2012)

\section{Additional information on J. Math. Phys.}

Journal Homepage: http://jmp.aip.org/

Journal Information: http://jmp.aip.org/about/about_the_journal

Top downloads: http://jmp.aip.org/features/most_downloaded

Information for Authors: http://jmp.aip.org/authors

\section{ADVERTISEMENT}

The most comprehensive support for physics in any mathematical software package World-leading tools for performing calculations in theoretical physics

Your work in Maple matches how you would write the problems and solutions by hand

- State-of-the-art environment for algebraic computations in physics

The only system with the ability to handle a wide range of physics computations as well as pencil-and-paper style input and textbook-quality display of results

- Access to Maple's full mathematical power, programming language, visualization routines, and document creation tools 


\title{
Global existence and nonrelativistic limit for the Vlasov-Maxwell-Chern-Simons system
}

\author{
Hyungjin Huh ${ }^{1, a)}$ and Ho Lee ${ }^{2, b)}$ \\ ${ }^{1}$ Department of Mathematics, Chung-Ang University, Seoul 156-756, South Korea \\ ${ }^{2}$ Max-Planck-Intitut für Gravitationsphysik, Albert-Einstein-Institut, Am Mühlenberg 1, \\ 14476 Potsdam, Germany
}

(Received 14 August 2012; accepted 25 September 2012; published online 22 October 2012)

The Vlasov-Maxwell-Chern-Simons system is studied. Global existence of classical solutions is proved, and then its nonrelativistic limit is investigated. When the speed of light tends to infinity, it is proved that solutions of the Vlasov-Maxwell-ChernSimons system converge to solutions of the Vlasov-Yukawa system in a suitable sense. (C) 2012 American Institute of Physics. [http://dx.doi.org/10.1063/1.4759129]

\section{INTRODUCTION}

As one of the simplest gauge theories, one can consider Maxwell's theory of electromagnetism. This theory can describe many electromagnetic phenomena very precisely, but a different type of gauge theory can also be used when electromagnetic phenomena are confined to a three (one temporal and two spatial) dimensional spacetime. The Chern-Simons theory ${ }^{6}$ is a gauge theory which is available in three-dimensional spacetime. This theory can describe many interesting phenomena such as the quantum Hall effect ${ }^{23,26}$ and high- $T_{c}$ superconductivity. ${ }^{22}$ The most interesting aspect of the Chern-Simons theory is that it provides mass for the gauge field without breaking the gauge symmetry. Hence, when it comes to three-dimensional cases, one may hope to find many interesting properties intrinsic to its dimensionality by considering the Chern-Simons theory in place of the Maxwell theory. A lot of mathematical works for the Chern-Simons theory ${ }^{3,4,14,18,25}$ have been found for last many years, and for more information we refer to Ref. 7.

In this paper, a system of partial differential equations which can describe an interaction between the Maxwell-Chern-Simons electromagnetic fields and the Vlasov matter is studied. The corresponding system of equations without the Chern-Simons term is known as the Vlasov-Maxwell system, and this system of equations has been studied for many years. There are many references (for instance, see Ref. 8 and the references therein), and the global existence problem has been fully analysed in lower dimensions. ${ }^{9-13}$ By considering the Chern-Simons term additionally to the Vlasov-Maxwell system, we will find that an interesting property of the Chern-Simons theory applies to a kinetic model. The mass generation property will be considered through the nonrelativistic limit problem in Sec. IV. Concerning this problem, some remarks will be given in Sec. I A.

The Lagrangian for the Maxwell-Chern-Simons electrodynamics is given as follows:

$$
\mathcal{L}=-\frac{1}{4} F_{\mu \nu} F^{\mu \nu}+\frac{\kappa}{2} \epsilon^{\mu \nu \rho} A_{\mu} \partial_{\nu} A_{\rho}-J_{\mu} A^{\mu},
$$

where all the indices run from 0 to 2 , and $\epsilon^{\mu \nu \rho}$ is the skew symmetric tensor with $\epsilon^{012}=1$. The three terms above correspond to the Maxwell term, the Chern-Simons term, and an interaction with matter coupled, respectively. The coupling constant $\kappa$ will be assumed to be unity, and $J_{\mu}$ is an electric current, which will be given by the Vlasov matter in this paper. Equations of fields for (1.1)

\footnotetext{
a)huh@cau.ac.kr.

b)ho.lee@aei.mpg.de.
} 
are given as follows:

$$
\partial_{\mu} F^{\mu \nu}+\frac{1}{2} \epsilon^{\nu \alpha \beta} F_{\alpha \beta}=J^{\nu} .
$$

To get equations for electromagnetic fields $E \in \mathbb{R}^{2}$ and $B \in \mathbb{R}^{1}$, we write $B=F_{21}$ and $E_{j}=F_{0 j}$ for $j=1,2$, and consequently Eqs. (1.3)-(1.5) together with the Bianchi identity (1.6) are obtained. On the other hand, for given electromagnetic fields the Vlasov equation can be written by (1.2) as in the two-dimensional Vlasov-Maxwell case. ${ }^{12,13}$ A system of equations is now obtained:

$$
\begin{gathered}
\partial_{t} f+\hat{v} \cdot \nabla_{x} f+\left(E_{1}+c^{-1} \hat{v}_{2} B, E_{2}-c^{-1} \hat{v}_{1} B\right) \cdot \nabla_{v} f=0, \\
\partial_{x_{1}} E_{1}+\partial_{x_{2}} E_{2}-B=\rho, \\
c^{-1} \partial_{t} E_{1}-\partial_{x_{2}} B+E_{2}=-c^{-1} j_{1}, \\
c^{-1} \partial_{t} E_{2}+\partial_{x_{1}} B-E_{1}=-c^{-1} j_{2}, \\
c^{-1} \partial_{t} B=\partial_{x_{2}} E_{1}-\partial_{x_{1}} E_{2} .
\end{gathered}
$$

The electric current has been written in the usual notation $\rho=J^{0}$ and $j_{i}=J^{i}$ for $i=1,2$, which are called the charge and current densities, respectively. The charge and current densities $\rho$ and $j$ are then induced from the distribution function $f$ in the following ways:

$$
\rho=4 \pi \int_{\mathbb{R}^{2}} f(v) d v, \quad j_{i}=4 \pi \int_{\mathbb{R}^{2}} \hat{v}_{i} f(v) d v .
$$

Note that since $f$ is a function of $t \geq 0, x \in \mathbb{R}^{2}$, and $v \in \mathbb{R}^{2}, \rho$ and $j$, like the field components $E$ and $B$, are functions of $t$ and $x$. In the above equations $\hat{v}$ denotes a relativistic velocity corresponding to a momentum $v$ :

$$
\hat{v}=\frac{v}{\sqrt{1+|v|^{2} / c^{2}}}
$$

In this paper, we will study the system of Eqs. (1.2)-(1.7), and let us call this system of equations the Vlasov-Maxwell-Chern-Simons (VMCS) system.

\section{A. Some remarks}

In this part, we briefly review the equations of the fields (1.3)-(1.6). When $\rho$ and $j$ are given, Eqs. (1.3)-(1.6) constitute an inhomogeneous linear system of equations, hence explicit formulae of solutions can be obtained. We first take summation of $t$ derivative of (1.4) multiplied by $c^{-1}$ and $x_{2}$ derivative of (1.6), and then subtract Eq. (1.5) and $x_{1}$ derivative of (1.3) from it. Then we obtain a Klein-Gordon type equation for $E_{1}$ field:

$$
c^{-2} \partial_{t}^{2} E_{1}-\Delta E_{1}+E_{1}=-\partial_{x_{1}} \rho-c^{-2} \partial_{t} j_{1}+c^{-1} j_{2} .
$$

By similar calculations, we obtain Klein-Gordon type equations for $E_{2}$ and $B$ fields:

$$
\begin{gathered}
c^{-2} \partial_{t}^{2} E_{2}-\Delta E_{2}+E_{2}=-\partial_{x_{2}} \rho-c^{-2} \partial_{t} j_{2}-c^{-1} j_{1}, \\
c^{-2} \partial_{t}^{2} B-\Delta B+B=-c^{-1} \partial_{x_{2}} j_{1}+c^{-1} \partial_{x_{1}} j_{2}-\rho .
\end{gathered}
$$

In other words, the VMCS system can be considered as a system of the Vlasov equation and KleinGordon type equations. We can find some references on these types of equations, ${ }^{17,19,20}$ but there were three-dimensional cases considered. Unlike three-dimensional cases, the fundamental solution of the two-dimensional Klein-Gordon equation is much similar to that of the linear wave equation. The fundamental solution of the Klein-Gordon equation has the following form:

$$
\frac{1}{2 \pi c} \frac{\cos \sqrt{c^{2} t^{2}-|x|^{2}}}{\sqrt{c^{2} t^{2}-|x|^{2}}} \chi_{B_{c t}(x)} \text {. }
$$


On the other hand, as for the Vlasov equation the most well-known equation is the VlasovMaxwell (VM) system. The Maxwell equations in the VM system are written as linear wave equations instead of (1.8)-(1.10):

$$
\begin{gathered}
c^{-2} \partial_{t}^{2} E-\Delta E=-\nabla_{x} \rho-c^{-2} \partial_{t} j, \\
c^{-2} \partial_{t}^{2} B-\Delta B=-c^{-1} \partial_{x_{2}} j_{1}+c^{-1} \partial_{x_{1}} j_{2},
\end{gathered}
$$

and it is well-known that the fundamental solution of two-dimensional wave equation has the following form:

$$
\frac{1}{2 \pi c} \frac{1}{\sqrt{c^{2} t^{2}-|x|^{2}}} \chi_{B_{c t}(x)} .
$$

By comparing (1.11) with (1.14) with the fact that the additional inhomogeneous terms in (1.8)-(1.10), which do not appear in (1.12) and (1.13), have no derivatives, we can expect that behaviors of solutions to the VMCS system are similar to those of the two-dimensional VM system.

The similarity between (1.11) and (1.14) helps to prove global existence of solutions to the VMCS system by applying some known results on the VM system. However, we will see that the VMCS system behaves in a different way when the speed of light $c$ tends to infinity, hence its nonrelativistic limit problem will be considerably nontrivial. On a formal level, it can be observed that Eqs. (1.8)-(1.10) converge to the following Yukawa type equations in nonrelativistic limit:

$$
-\Delta E+E=-\nabla_{x} \rho, \quad-\Delta B+B=-\rho,
$$

while Eqs. (1.12) and (1.13) converge to the following Poisson equations:

$$
-\Delta E=-\nabla_{x} \rho, \quad-\Delta B=0 .
$$

We can see that solutions of the former equations have fast decaying properties due to the ChernSimons term. Moreover, the magnetic field does not disappear in nonrelativistic limit, but it plays a role as a potential of the electric field, i.e., $E=\nabla_{x} B$. This is the main difference between the VMCS and the VM cases, and detailed proof for this interesting property will be given in Sec. IV.

\section{B. Notations and preliminaries}

\section{Notations}

In the VMCS system (1.2)-(1.7), mass and charge of particles are assumed to be unity, while the speed of light $c$ is not, because the constant $c$ will be used as a parameter in nonrelativistic limit of the VMCS system in Sec. IV. Without loss of generality, we assume that $c \geq 1$. Generic positive constants are denoted by $D$, which may vary line to line. Similarly, $C(t)$ denotes a continuous nondecreasing function, which may also vary from line to line.

A function $g=g(t, y)$ is said to be $g=h+O(\varepsilon)$ on a time interval $I$, if $g$ satisfies

$$
|g(t, y)-h(t, y)| \leq D \varepsilon,
$$

for any $t \in I$ and $y \in \mathbb{R}^{2}$ (or $y \in \mathbb{R}^{2} \times \mathbb{R}^{2}$ ). Here the constant $D$ does not depend on $\varepsilon$.

In two-dimensional cases, the Lorentz force is written as in (1.2). For simplicity, a twodimensional vector field $F=F(t, x, v)$ will denote the Lorentz force in some places.

$$
F=\left(E_{1}+c^{-1} \hat{v}_{2} B, E_{2}-c^{-1} \hat{v}_{1} B\right) .
$$

\section{Preliminaries}

In this part, we collect some theorems and formulae without proofs. The proofs can be found in references quoted below. 
As for the Vlasov equation (1.2), we can define characteristic curves:

$$
\begin{aligned}
& \partial_{s} X(s ; t, x, v)=V(s ; t, x, v) / \sqrt{1+|V(s)|^{2} / c^{2}}, \quad X(t ; t, x, v)=x, \\
& \partial_{s} V(s ; t, x, v)=F(s, X(s), V(s)), \quad V(t ; t, x, v)=v,
\end{aligned}
$$

where $X(s)$ and $V(s)$ denote $X(s ; t, x, v)$ and $V(s ; t, x, v)$, respectively. Along the characteristic curves, the Vlasov equation is written as follows:

$$
\partial_{s}[f(s, X(s), V(s))]=0,
$$

which implies that

$$
f(t, x, v)=f(0, X(0), V(0)) \quad \text { and } \quad f(t, x, v) \leq\|f(0)\|_{L_{x, v}^{\infty}} .
$$

Throughout the paper, it will be assumed that initial data $f(0)$ is nonnegative and has compact support both in $x$ and $v$, say $\{|x|,|v| \leq R\}$ for some $R$. If $(f, E, B)$ is a classical solution to the VMCS system, then the characteristic curves are well defined and have a unique classical solution. Moreover, the following quantity is also well defined:

$$
P(t)=\sup \left\{|v|: f(s, x, v) \neq 0, s \in[0, t], x \in \mathbb{R}^{2}, v \in \mathbb{R}^{2}\right\},
$$

which is a nondecreasing continuous function, and we obtain the following properties:

$$
f(t, x, v)=0, \quad \text { if } \quad|x| \geq R+t P(t) \quad \text { or } \quad|v| \geq P(t) .
$$

In other words, $f(t)$ for each $t$ has compact support both in $x$ and $v$. This property will be repeatedly used throughout the paper. We refer to Refs. 8, 12,13, and 21, and the references therein for detailed discussions on the Vlasov equation.

We will show that solutions to the VMCS system converge to solutions to the Vlasov-Yukawa (VY) system, where force is given by the Yukawa potential:

$$
F=\nabla_{x} \phi, \quad-\Delta \phi+\phi=-\rho,
$$

where $\rho$ is a charge density given by the Vlasov equation. The VY system has been recently studied in Refs. 2, 15, and 16, and its two-dimensional case in Ref. 5. In the present paper, we have to deal with the Yukawa type equation, so we need some preliminaries. In two dimensions, the Yukawa equation above has the following fundamental solution:

$$
\frac{1}{2 \pi} K_{0}(|x|)
$$

where $K_{0}$ is the modified Bessel function of the second kind of order zero. We refer to Ref. 24 for various theorems and formulae concerning the Bessel functions. In this paper, we need the following properties of the modified Bessel functions of the second kind.

The following properties are found in Ref. 1 (see p. 375 and p. 378, Chapter 9). When $n$ is fixed and $z \rightarrow 0$, we have

$$
K_{0}(z) \sim-\log z, \quad K_{n}(z) \sim \frac{\Gamma(n)}{2}\left(\frac{2}{z}\right)^{n} \quad \text { for } \quad n>0,
$$

where $\Gamma$ is the gamma function, which is defined as $\Gamma(n)=(n-1)$ ! for integer $n>0$. On the other hand, when $n$ is fixed, $|z|$ is large, and $\mu=4 n^{2}$, we have

$$
K_{n}(z) \sim \sqrt{\frac{\pi}{2 z}} e^{-z}\left(1+\frac{\mu-1}{8 z}+\frac{(\mu-1)(\mu-9)}{2 !(8 z)^{2}}+\frac{(\mu-1)(\mu-9)(\mu-25)}{3 !(8 z)^{3}}+\cdots\right)
$$

for $|\arg z|<\frac{3}{2} \pi$. In this paper, we encounter only $n=0,1$ cases with real numbers $z>0$. Then, in the above expansion, the remainder after $k$ terms does not exceed absolute value of the $(k+1)$ th term for $k \geq 1$ (see p. 378, Chapter 9 of Ref. 1). In other words, the above expansion can be written as

$$
K_{n}(z) \sim \sqrt{\frac{\pi}{2 z}} e^{-z}\left(1+O\left(z^{-1}\right)\right) \text { for } \quad z \gg 1
$$


With the asymptotic behaviors near the origin (1.16) and at infinity (1.17), we obtain the following lemma.

Lemma 1.1: Let $g$ be a continuous function with compact support $\left\{x \in \mathbb{R}^{2}:|x| \leq R\right\}$, and consider the following quantity:

$$
G(x)=\int_{\mathbb{R}^{2}} K_{n}(|x-y|) g(y) d y \quad \text { for } \quad n=0,1 .
$$

Then, $G$ satisfies $|G(x)| \leq D e^{-\frac{1}{2}|x|}$ for some positive constant $D$.

Proof: Note that $K_{0}$ and $K_{1}$ are integrable on $\mathbb{R}^{2}$ thanks to (1.16) and (1.17). Hence, it is easy to show that $|G(x)| \leq D\|g\|_{L_{x}^{\infty}}$ for some $D$. When $x$ is large, say $|x| \geq 2 R, K_{n}$ satisfies

$$
K_{n}(|x-y|) \leq D e^{-|x|+R} \leq D e^{-\frac{1}{2}|x|} .
$$

Since $g$ is also integrable, we obtain the desired result.

The following formula, which is called Basset's integral, will be crucially used to obtain nonrelativistic limit of the VMCS system. Detailed discussions on Basset's integral are found in Ref. 24 (see p. 172, Chapter 6):

$$
K_{n}(m x)=\frac{\Gamma\left(n+\frac{1}{2}\right)(2 m)^{n}}{x^{n} \Gamma\left(\frac{1}{2}\right)} \int_{0}^{\infty} \frac{\cos (x u)}{\left(m^{2}+u^{2}\right)^{n+1 / 2}} d u,
$$

which is valid for $\Re\left(n+\frac{1}{2}\right) \geq 0, x>0$, and $|\arg m|<\frac{1}{2} \pi$. In this paper, we only consider $n=0,1$ cases with $m=1$ :

$$
K_{0}(x)=\int_{0}^{\infty} \frac{\cos (x u)}{\sqrt{1+u^{2}}} d u
$$

and

$$
K_{1}(x)=\frac{1}{x} \int_{0}^{\infty} \frac{\cos (x u)}{\left(1+u^{2}\right)^{3 / 2}} d u .
$$

We also need a different form of (1.18). In order to derive it, we need the following properties, which are found in Ref. 24 (see p. 79, Chapter 3):

$$
\left(\frac{d}{x d x}\right)^{m}\left[x^{n} K_{n}(x)\right]=(-1)^{m} x^{n-m} K_{n-m}(x) \text { and } \frac{d}{d x} K_{0}(x)=-K_{1}(x) .
$$

In this paper, we use only $n=m=1$ case in the formula above left:

$$
\frac{d}{d x}\left[x K_{1}(x)\right]=-x K_{0}(x) \text { and } \frac{d}{d x} K_{0}(x)=-K_{1}(x) .
$$

We now use the above formulae (1.18)-(1.20) to obtain the following lemma.

Lemma 1.2: The modified Bessel function of the second kind of order zero can be written as

$$
K_{0}(x)=\int_{0}^{\infty} \frac{u \sin (x u)}{x\left(1+u^{2}\right)^{3 / 2}} d u .
$$

Proof: This is a direct consequence of the formula (1.19) with the left one in (1.20):

$$
-x K_{0}(x)=\frac{d}{d x}\left[x K_{1}(x)\right]=\frac{d}{d x}\left[\int_{0}^{\infty} \frac{\cos (x u)}{\left(1+u^{2}\right)^{3 / 2}} d u\right]=-\int_{0}^{\infty} \frac{u \sin (x u)}{\left(1+u^{2}\right)^{3 / 2}} d u,
$$

and this completes the proof.

Remark 1.1: In most estimations, we take absolute values on integrals considered. If we simply take the absolute value on (1.18), we encounter an integration which does not converge, i.e., integration of $\frac{1}{\sqrt{1+u^{2}}}$. However, this problem is overcome by the above lemma. When we use the 
formula for $K_{0}$ given in the above lemma instead of (1.18), we get an integration of $\frac{u}{\left(1+u^{2}\right)^{3 / 2}}$, which does converge. This lemma will be crucially used in estimations of the magnetic field of the VMCS system.

Lemma 1.3: Let $g$ be a $C_{c}^{2}$ function with compact support $\left\{x \in \mathbb{R}^{2}:|x| \leq R\right\}$, and consider the Yukawa equation:

$$
-\Delta \phi+\phi=g .
$$

Then, $\phi$ satisfies $\left|\nabla_{x}^{\alpha} \phi(x)\right| \leq D e^{-\frac{1}{2}|x|}$ for multi-indices $|\alpha| \leq 3$ and some positive constant $D$.

Proof: The proof is a direct consequence of Lemma 1.1 with the second formula of (1.20). We first note that $\phi$ is written as

$$
\phi(x)=\frac{1}{2 \pi} \int_{\mathbb{R}^{2}} K_{0}(|x-y|) g(y) d y .
$$

Since $g$ is twice continuously differentiable, we have for $|\alpha|=3$ :

$$
\partial_{i} \partial_{j} \partial_{k} \phi(x)=-\frac{1}{2 \pi} \int_{\mathbb{R}^{2}} K_{1}(|x-y|) \frac{\left(x_{i}-y_{i}\right)}{|x-y|} \partial_{j} \partial_{k} g(y) d y,
$$

where we used (1.20), and Lemma 1.1 gives the desired result. The other cases, $|\alpha|<3$, are also clear, and this completes the proof.

\section{Organization of the paper}

This paper is organized as follows. We first derive representations of the fields in Sec. II. This representation formulae will be used throughout this paper. With these formulae we obtain global existence of classical solutions to the VMCS system in Sec. III. Due to the similarity between (1.11) and (1.14), the argument of global existence is much similar to that of Refs. 12 and 13, where global existence of the VM system was proved in two dimensions. In Sec. IV, we study nonrelativistic limit of the VMCS system. In Ref. 21, it was proved that the two-dimensional VM system converges to the two-dimensional Vlasov-Poisson system. We apply a part of the argument of Ref. 21 to the VMCS system, then we will see that the VMCS system converges to a different type of Vlasov equation, rather than the VP system, which is called the Vlasov-Yukawa system.

\section{REPRESENTATIONS OF THE FIELDS}

In this section, we apply the main idea of Ref. 9 to the VMCS case in order to obtain representations of the fields. The usual $t$ and $x$ derivatives are decomposed into linear combinations of two types of differential operators $S$ and $T$ :

$$
S=\partial_{t}+\hat{v} \cdot \nabla_{x} \quad \text { and } \quad T_{k}=\frac{1}{\sqrt{1-|\xi|^{2}}}\left(\partial_{x_{k}}-c^{-1} \xi_{k} \partial_{t}\right), \quad k=1,2,
$$

where a two-dimensional vector $\xi$ is defined by

$$
\xi=\frac{y-x}{c(t-\tau)} .
$$

We follow the calculations of Refs. 12,13, and 21, where the representations of the Maxwell fields were recovered to the case of arbitrary $c$ in Ref. 21, and we obtain the following lemma.

Lemma 2.1: Suppose that $(f, E, B)$ is a continuously differentiable solution of the VMCS system (1.2)-(1.7), where f has compact support in $x$ and $v$ for each $t$. Then, they are represented as follows:

$$
\begin{aligned}
& E_{k}=\tilde{E}_{k}^{F}+\tilde{E}_{k}^{T_{1}}+\tilde{E}_{k}^{T_{2}}+E_{k}^{S}+E_{k}^{T}+E_{k}^{N}, \quad k=1,2, \\
& B=\tilde{B}^{F}+\tilde{B}^{T_{1}}+\tilde{B}^{T_{2}}+B^{S}+B^{T}+B^{N},
\end{aligned}
$$


which are defined by

$$
\begin{aligned}
& E_{k}^{S}=\iiint \frac{\cos \sqrt{c^{2}(t-\tau)^{2}-|x-y|^{2}}}{c \sqrt{c^{2}(t-\tau)^{2}-|x-y|^{2}}}(F f)(\tau, y, v) \cdot \nabla_{v}\left[\frac{-2\left(c \xi_{k}+\hat{v}_{k}\right)}{1+c^{-1} \hat{v} \cdot \xi}\right] d v d y d \tau, \\
& E_{k}^{T}=\iiint \frac{\cos \sqrt{c^{2}(t-\tau)^{2}-|x-y|^{2}}}{c^{3}(t-\tau) \sqrt{c^{2}(t-\tau)^{2}-|x-y|^{2}}} f(\tau, y, v)\left(\frac{-2\left(c^{2}-|\hat{v}|^{2}\right)\left(c \xi_{k}+\hat{v}_{k}\right)}{\left(1+c^{-1} \hat{v} \cdot \xi\right)^{2}}\right) d v d y d \tau \\
& E_{1}^{N}=\iiint \frac{\cos \sqrt{c^{2}(t-\tau)^{2}-|x-y|^{2}}}{\sqrt{c^{2}(t-\tau)^{2}-|x-y|^{2}}}\left(2 \hat{v}_{2} f\right)(\tau, y, v) d v d y d \tau \\
& E_{2}^{N}=\iiint \frac{\cos \sqrt{c^{2}(t-\tau)^{2}-|x-y|^{2}}}{\sqrt{c^{2}(t-\tau)^{2}-|x-y|^{2}}}\left(-2 \hat{v}_{1} f\right)(\tau, y, v) d v d y d \tau, \\
& B^{S}=\iiint \frac{\cos \sqrt{c^{2}(t-\tau)^{2}-|x-y|^{2}}}{c \sqrt{c^{2}(t-\tau)^{2}-|x-y|^{2}}}(F f)(\tau, y, v) \cdot \nabla_{v}\left[\frac{2\left(\xi_{1} \hat{v}_{2}-\xi_{2} \hat{v}_{1}\right)}{1+c^{-1} \xi \cdot \hat{v}}\right] d v d y d \tau, \\
& B^{T}=\iiint \frac{\cos \sqrt{c^{2}(t-\tau)^{2}-|x-y|^{2}}}{c^{3}(t-\tau) \sqrt{c^{2}(t-\tau)^{2}-|x-y|^{2}}} f(\tau, y, v)\left(\frac{2\left(\xi_{1} \hat{v}_{2}-\xi_{2} \hat{v}_{1}\right)\left(c^{2}-|\hat{v}|^{2}\right)}{\left(1+c^{-1} \hat{v} \cdot \xi\right)^{2}}\right) d v d y d \tau, \\
& B^{N}=\iiint \frac{\cos \sqrt{c^{2}(t-\tau)^{2}-|x-y|^{2}}}{\sqrt{c^{2}(t-\tau)^{2}-|x-y|^{2}}}(-2 c f)(\tau, y, v) d v d y d \tau
\end{aligned}
$$

where all the integration domains are $\left\{(\tau, y, v) \in[0, t] \times \mathbb{R}^{2} \times \mathbb{R}^{2}:|x-y| \leq c(t-\tau)\right\}$, and $F$ denotes

$$
F=\left(E_{1}+c^{-1} \hat{v}_{2} B, E_{2}-c^{-1} \hat{v}_{1} B\right) .
$$

The other terms $\tilde{E}_{k}^{F}$ and $\tilde{B}^{F}$ depend only on initial data of $E$ and $B$, while $\tilde{E}_{k}^{T_{1}}, \tilde{E}_{k}^{T_{2}}, \tilde{B}^{T_{1}}$, and $\tilde{B}^{T_{2}}$ depend only on initial data of $f$. They are defined by

$$
\begin{aligned}
& \tilde{E}_{k}^{F}=\frac{1}{2 \pi c}\left(\int \frac{\cos \sqrt{c^{2} t^{2}-|x-y|^{2}}}{\sqrt{c^{2} t^{2}-|x-y|^{2}}} \partial_{t} E_{k}(0, y) d y+\frac{\partial}{\partial t}\left[\int \frac{\cos \sqrt{c^{2} t^{2}-|x-y|^{2}}}{\sqrt{c^{2} t^{2}-|x-y|^{2}}} E_{k}(0, y) d y\right]\right), \\
& \tilde{B}^{F}=\frac{1}{2 \pi c}\left(\int \frac{\cos \sqrt{c^{2} t^{2}-|x-y|^{2}}}{\sqrt{c^{2} t^{2}-|x-y|^{2}}} \partial_{t} B(0, y) d y+\frac{\partial}{\partial t}\left[\int \frac{\cos \sqrt{c^{2} t^{2}-|x-y|^{2}}}{\sqrt{c^{2} t^{2}-|x-y|^{2}}} B(0, y) d y\right]\right),
\end{aligned}
$$

where the integration domains are $\{y:|x-y| \leq c t\}$, and

$$
\begin{aligned}
& \tilde{E}_{1}^{T_{1}}=\left.\iint \frac{-c^{2}-c \xi_{2} \hat{v}_{2}+\hat{v}_{1}^{2}}{c^{2}(t-\tau)\left(1+c^{-1} \xi \cdot \hat{v}\right)} \cos \sqrt{c^{2}(t-\tau)^{2}-|x-y|^{2}} \frac{2 c^{-1} \xi_{1} f(0, y, v)}{\sqrt{1-|\xi|^{2}}}\right|_{\tau=0} d y d v, \\
& \tilde{E}_{1}^{T_{2}}=\left.\iint \frac{c \xi_{1} \hat{v}_{2}+\hat{v}_{1} \hat{v}_{2}}{c^{2}(t-\tau)\left(1+c^{-1} \xi \cdot \hat{v}\right)} \cos \sqrt{c^{2}(t-\tau)^{2}-|x-y|^{2}} \frac{2 c^{-1} \xi_{2} f(0, y, v)}{\sqrt{1-|\xi|^{2}}}\right|_{\tau=0} d y d v, \\
& \tilde{E}_{2}^{T_{1}}=\left.\iint \frac{c \xi_{2} \hat{v}_{1}+\hat{v}_{1} \hat{v}_{2}}{c^{2}(t-\tau)\left(1+c^{-1} \xi \cdot \hat{v}\right)} \cos \sqrt{c^{2}(t-\tau)^{2}-|x-y|^{2}} \frac{2 c^{-1} \xi_{1} f(0, y, v)}{\sqrt{1-|\xi|^{2}}}\right|_{\tau=0} d y d v,
\end{aligned}
$$




$$
\begin{aligned}
& \tilde{E}_{2}^{T_{2}}=\left.\iint \frac{-c^{2}-c \xi_{1} \hat{v}_{1}+\hat{v}_{2}^{2}}{c^{2}(t-\tau)\left(1+c^{-1} \xi \cdot \hat{v}\right)} \cos \sqrt{c^{2}(t-\tau)^{2}-|x-y|^{2}} \frac{2 c^{-1} \xi_{2} f(0, y, v)}{\sqrt{1-|\xi|^{2}}}\right|_{\tau=0} d y d v, \\
& \tilde{B}^{T_{1}}=\left.\iint \frac{\hat{v}_{2}+c^{-1} \xi_{2}|\hat{v}|^{2}}{c(t-\tau)\left(1+c^{-1} \xi \cdot \hat{v}\right)} \cos \sqrt{c^{2}(t-\tau)^{2}-|x-y|^{2}} \frac{2 c^{-1} \xi_{1} f(0, y, v)}{\sqrt{1-|\xi|^{2}}}\right|_{\tau=0} d y d v, \\
& \tilde{B}^{T_{2}}=\left.\iint \frac{-\hat{v}_{1}-c^{-1} \xi_{1}|\hat{v}|^{2}}{c(t-\tau)\left(1+c^{-1} \xi \cdot \hat{v}\right)} \cos \sqrt{c^{2}(t-\tau)^{2}-|x-y|^{2}} \frac{2 c^{-1} \xi_{2} f(0, y, v)}{\sqrt{1-|\xi|^{2}}}\right|_{\tau=0} d y d v,
\end{aligned}
$$

where all the integration domains are $\{y:|x-y| \leq c t\} \times \mathbb{R}^{2}$.

Proof: The calculations are almost same as in Refs. 12 and 21, so we only present a sketch of it. We first note that $\tilde{E}_{k}^{F}$ and $\tilde{B}^{F}$ are apparently the homogeneous parts of the solutions. In order to use the fundamental solution (1.11), we multiply Eqs. (1.8)-(1.10) by $c^{2}$, and then obtain the formula for the fields. For instance, the $B$ field is written as follows:

$$
B=\tilde{B}^{F}+\frac{1}{2 \pi c} \int_{0}^{t} \int_{|x-y| \leq c(t-\tau)} \frac{\cos \sqrt{c^{2}(t-\tau)^{2}-|x-y|^{2}}}{\sqrt{c^{2}(t-\tau)^{2}-|x-y|^{2}}}\left(c \partial_{x_{1}} j_{2}-c \partial_{x_{2}} j_{1}-c^{2} \rho\right)(\tau, y) d y d \tau .
$$

We remind that the charge density $\rho$ is defined by (1.7), and then the $B^{N}$ term appears from the last term, $-c^{2} \rho$, in the above integral. All the other terms come out from $c \partial_{x_{1}} j_{2}-c \partial_{x_{2}} j_{1}$ when we decompose the partial derivatives into linear combinations of $S$ and $T$. More explicitly, time, and spatial derivatives are decomposed as follows:

$$
\begin{aligned}
\partial_{t} & =\frac{1}{1+c^{-1} \xi \cdot \hat{v}}\left(S-\sqrt{1-|\xi|^{2}}(\hat{v} \cdot T)\right), \\
\partial_{x_{1}} & =\frac{1}{1+c^{-1} \xi \cdot \hat{v}}\left(c^{-1} \xi_{1} S+\sqrt{1-|\xi|^{2}}\left(\left(1+c^{-1} \xi_{2} \hat{v}_{2}\right) T_{1}-c^{-1} \xi_{1} \hat{v}_{2} T_{2}\right)\right), \\
\partial_{x_{2}} & =\frac{1}{1+c^{-1} \xi \cdot \hat{v}}\left(c^{-1} \xi_{2} S+\sqrt{1-|\xi|^{2}}\left(\left(1+c^{-1} \xi_{1} \hat{v}_{1}\right) T_{2}-c^{-1} \xi_{2} \hat{v}_{1} T_{1}\right)\right) .
\end{aligned}
$$

Note that since $S f=-F \cdot \nabla_{v} f=-\nabla_{v} \cdot(F f)$, we can use integration by parts with respect to $v$. However, there is no $v$ variable inside the cosine function, so the integration by parts with respect to $v$ does not affect the cosine function, and then we obtain $E_{k}^{S}$ and $B^{S}$ terms.

As for the $T$ terms, we note that

$$
T_{1} g(\tau, y)=\nabla_{\tau, y} \cdot\left[\frac{\left(-c^{-1} \xi_{1}, 1,0\right)}{\sqrt{1-|\xi|^{2}}} g(\tau, y)\right], \quad T_{2} g(\tau, y)=\nabla_{\tau, y} \cdot\left[\frac{\left(-c^{-1} \xi_{2}, 0,1\right)}{\sqrt{1-|\xi|^{2}}} g(\tau, y)\right]
$$

for any differentiable function $g$. Hence, we can take integration by parts with respect to $(\tau, y)$. By integration by parts on the domain $\left\{(\tau, y) \in[0, t] \times \mathbb{R}^{2}:|x-y| \leq c(t-\tau)\right\}$, we obtain $B^{T}$ together with two kinds of boundary terms; one from the boundary $\{|x-y|=c(t-\tau)\}$ and the other one from the other boundary $\{\tau=0\}$. Then, we obtain $\tilde{B}^{T_{1}}$ and $\tilde{B}^{T_{2}}$ from the second boundary term. We can see that the first boundary term vanishes by the same argument as in Ref. 21, because the cosine function, which is the only difference of (1.11) from (1.14), satisfies the following calculation:

$$
\begin{aligned}
& \nabla_{\tau, y}\left[\cos \sqrt{c^{2}(t-\tau)^{2}-|x-y|^{2}}\right] \cdot\left(-c^{-1} \xi_{1}, 1,0\right) \\
& =-\sin \sqrt{c^{2}(t-\tau)^{2}-|x-y|^{2}}\left(-c^{-1} \xi_{1} \frac{c^{2}(\tau-t)}{\sqrt{c^{2}(t-\tau)^{2}-|x-y|^{2}}}-\frac{y_{1}-x_{1}}{\sqrt{c^{2}(t-\tau)^{2}-|x-y|^{2}}}\right) \\
& =0,
\end{aligned}
$$


and similarly the cosine function does not play a role in the calculation for $T_{2}$ term. After lengthy calculations we then obtain the desired representation formulae for $E$ and $B$.

Remark 2.1: The representations for $E$ and $B$ fields in Lemma 2.1 are almost same with the results of Refs. 12 and 21 in the Maxwell cases (see Appendix A of Ref. 21 for arbitrary c cases). Note that we have only two different points from the results of Refs. 12 and 21. The first one is that cosine functions appear in the integrals of the representations. This is due to the fundamental solution (1.11) for the Klein-Gordon equation. However, the cosine functions are clearly bounded and do not make serious problems thanks to (2.3). The second point of difference is that we have additional inhomogeneous terms, from which $E^{N}$ and $B^{N}$ are obtained. However, these additional terms do not have derivatives, hence which can be controlled by suitable estimations in Secs. III and IV.

\section{EXISTENCE OF CLASSICAL SOLUTIONS}

In this section, we show that classical solutions to the VMCS system exist globally in time. Global existence of solutions for the two-dimensional VM case was proved in Refs. 12 and 13 . Basically the same arguments are applied to the VMCS case to obtain global existence of solutions. In this section, the speed of light will be assumed to be unity. We denote nondecreasing functions by $C(t)$, which may vary line to line.

We first prove that if there exists a nondecreasing function $C(t)$ such that the momentum support of $f$ is bounded by $C(t)$, then the fields can be controlled.

Lemma 3.1: Let $(f, E, B)$ be a continuously differentiable solution of the VMCS system (1.2)-(1.7), where $f$ has compact support in $x$ and $v$ for each $t$. Suppose that initial data are finite, i.e.,

$$
\left\|\nabla_{x, v}^{\alpha} f(0)\right\|_{L_{x, v}^{\infty}}+\left\|\nabla_{x}^{\beta} E(0)\right\|_{L_{x}^{\infty}}+\left\|\nabla_{x}^{\beta} B(0)\right\|_{L_{x}^{\infty}}<\infty \quad \text { for } \quad|\alpha| \leq 1 \quad \text { and } \quad|\beta| \leq 2,
$$

and there exists a nondecreasing function $C(t)$ such that

$$
f(t, x, v)=0 \quad \text { for } \quad|v| \geq C(t)
$$

Then, we have the following estimations:

$$
\sup _{0 \leq \tau \leq t}\left\{\|E(\tau)\|_{L_{x}^{\infty}}+\|B(\tau)\|_{L_{x}^{\infty}}\right\} \leq C(t)
$$

and

$$
\sup _{0 \leq \tau \leq t}\left\{\left\|\nabla_{x} E(\tau)\right\|_{L_{x}^{\infty}}+\left\|\nabla_{x} B(\tau)\right\|_{L_{x}^{\infty}}\right\} \leq C(t)\left(1+\ln ^{*}\left(t \sup _{0 \leq \tau \leq t}\left\|\nabla_{x, v} f(\tau)\right\|_{L_{x, v}^{\infty}}\right)\right),
$$

where the function $\ln *$ is defined by

$$
\ln ^{*}(x)=\max \{0, \ln (x)\} \quad \text { for } \quad x>0 .
$$

Proof: The lemma is proved by following the calculations of Ref. 12 and using the representations given in Lemma 2.1. We consider the $B^{N}$ term in Lemma 2.1:

$$
\begin{aligned}
\left|B^{N}\right| & =\left|\iiint \frac{\cos \sqrt{(t-\tau)^{2}-|x-y|^{2}}}{\sqrt{(t-\tau)^{2}-|x-y|^{2}}}(2 f)(\tau, y, v) d v d y d \tau\right| \\
& \leq C(t) \iiint_{|v| \leq C(t)} \frac{1}{\sqrt{(t-\tau)^{2}-|x-y|^{2}}} d v d y d \tau
\end{aligned}
$$


since $f(t, x, v)=0$ for $|v| \geq C(t)$. We proceed the estimation as follows:

$$
\begin{aligned}
\left|B^{N}\right| & \leq C(t) \iiint_{|v| \leq C(t)} \frac{1}{\sqrt{(t-\tau)^{2}-|x-y|^{2}}} d v d y d \tau \\
& \leq C(t) \int_{0}^{t} \int_{|x-y| \leq t-\tau} \frac{1}{\sqrt{(t-\tau)^{2}-|x-y|^{2}}} d y d \tau \\
& \leq C(t) \int_{0}^{t} t-\tau d \tau \\
& \leq C(t) .
\end{aligned}
$$

Then, $E_{1}^{N}$ and $E_{2}^{N}$ terms are similarly estimated. The other terms are estimated by the same calculations as in Ref. 12 because the cosine functions are bounded as

$$
\left|\cos \sqrt{(t-\tau)^{2}-|x-y|^{2}}\right| \leq 1
$$

and we obtain the first estimation for $E$ and $B$.

In order to obtain the second estimation for $\nabla_{x} E$ and $\nabla_{x} B$, we should take $x$ derivatives on the representations in Lemma 2.1. We first consider $B^{N}$ term:

$$
\begin{aligned}
\partial_{x_{1}} B^{N}= & \iiint \frac{\cos \sqrt{(t-\tau)^{2}-|x-y|^{2}}}{\sqrt{(t-\tau)^{2}-|x-y|^{2}}}\left(-2 \partial_{x_{1}} f\right)(\tau, y, v) d v d y d \tau \\
= & \iiint \frac{\cos \sqrt{(t-\tau)^{2}-|x-y|^{2}}}{\sqrt{(t-\tau)^{2}-|x-y|^{2}}} \frac{-2 \xi_{1}}{1+\xi \cdot \hat{v}} S f d v d y d \tau \\
& +\iiint \frac{\cos \sqrt{(t-\tau)^{2}-|x-y|^{2}}}{(t-\tau)(1+\xi \cdot \hat{v})}\left(-2\left(1+\xi_{2} \hat{v}_{2}\right) T_{1} f+2 \xi_{1} \hat{v}_{2} T_{2} f\right) d v d y d \tau \\
= & B^{N S}+B^{N T},
\end{aligned}
$$

where we used (2.1) for the $x$ derivative. Then, $B^{N S}$ and $B^{N T}$ are estimated separately:

$$
\begin{aligned}
\left|B^{N S}\right| & =\left|\iiint \frac{\cos \sqrt{(t-\tau)^{2}-|x-y|^{2}}}{\sqrt{(t-\tau)^{2}-|x-y|^{2}}} \nabla_{v}\left[\frac{-2 \xi_{1}}{1+\xi \cdot \hat{v}}\right] \cdot F f d v d y d \tau\right| \\
& \leq C(t) \iiint \frac{1}{\sqrt{(t-\tau)^{2}-|x-y|^{2}}}\left|\nabla_{v}\left[\frac{\xi_{1}}{1+\xi \cdot \hat{v}}\right]\right| f d v d y d \tau,
\end{aligned}
$$

where we used the first estimation of the lemma for $F$. Since $f(t, x, v)=0$ for $|v| \geq C(t)$, we can proceed the estimation as follows:

$$
\begin{aligned}
\left|B^{N S}\right| & \leq C(t) \iiint_{|v| \leq C(t)} \frac{1}{\sqrt{(t-\tau)^{2}-|x-y|^{2}}} d v d y d \tau \\
& \leq C(t) \int_{0}^{t} \int_{|x-y| \leq t-\tau} \frac{1}{\sqrt{(t-\tau)^{2}-|x-y|^{2}}} d y d \tau \\
& \leq C(t) .
\end{aligned}
$$


As for the $B^{N T_{1}}$ term, we use (2.2) and (2.3) to take integration by parts:

$$
\begin{aligned}
B^{N T_{1}}= & \iiint \frac{\cos \sqrt{(t-\tau)^{2}-|x-y|^{2}}}{(t-\tau)(1+\xi \cdot \hat{v})}\left(-2\left(1+\xi_{2} \hat{v}_{2}\right) T_{1} f\right) d v d y d \tau \\
= & \iiint \frac{\cos \sqrt{(t-\tau)^{2}-|x-y|^{2}}}{(t-\tau)(1+\xi \cdot \hat{v})}\left(-2\left(1+\xi_{2} \hat{v}_{2}\right)\right) \nabla_{(\tau, y)} \cdot\left[\frac{\left(-\xi_{1}, 1,0\right)}{\left.\sqrt{1-|\xi|^{2}} f\right] d v d y d \tau}\right. \\
= & \text { (boundary terms) } \\
& -\iiint \frac{\cos \sqrt{(t-\tau)^{2}-|x-y|^{2}}}{\sqrt{1-|\xi|^{2}}} \underbrace{\left.-\xi_{1}, 1,0\right) \cdot \nabla_{(\tau, y)}\left[\frac{-2\left(1+\xi_{2} \hat{v}_{2}\right)}{(t-\tau)(1+\xi \cdot \hat{v})}\right]}_{A} f d v d y d \tau,
\end{aligned}
$$

where the boundaries are $|x-y|=t-\tau$ and $\tau=0$. The second boundary term is easily estimated because $f$ depends only on initial data. On the first boundary, the integrand becomes singular, but which can be estimated by the same argument as in Ref. 12, for instance (2.10) at p. 337 of Ref. 12. The $A$ term in the integral above is estimated by a direct calculation as follows:

$$
|A| \leq C(t)(t-\tau)^{-2} \text { for }|v| \leq C(t)
$$

Hence, we obtain

$$
\begin{aligned}
\left|B^{N T_{1}}\right| & \leq C(t) \iiint \frac{f}{(t-\tau) \sqrt{(t-\tau)^{2}-|x-y|^{2}}} d v d y d \tau \\
& \leq C(t) \int_{0}^{t} \int_{|x-y| \leq t-\tau} \frac{1}{(t-\tau) \sqrt{(t-\tau)^{2}-|x-y|^{2}}} d y d \tau \\
& \leq C(t) \int_{0}^{t} d \tau \\
& \leq C(t) .
\end{aligned}
$$

By similar calculations, the $B^{N T_{2}}$ term is also estimated as $\left|B^{N T_{2}}\right| \leq C(t)$. For the other terms, i.e.,

$$
\partial_{x_{1}} \tilde{B}^{F}+\partial_{x_{1}} \tilde{B}^{T_{1}}+\partial_{x_{1}} \tilde{B}^{T_{2}}+\partial_{x_{1}} B^{S}+\partial_{x_{1}} B^{T},
$$

we can use the same argument as in Ref. 12 thanks to (2.3) and boundedness of the cosine functions. Hence, the calculations turn out to be much similar to those in Ref. 12, and we obtain the following inequality:

$$
\left|\partial_{x_{1}} B\right| \leq C(t)\left(1+\ln ^{*}\left(t \sup _{0 \leq \tau \leq t}\left\|\nabla_{x, v} f(\tau)\right\|_{L_{x, v}^{\infty}}\right)+\int_{0}^{t}\left\|\nabla_{x} E(\tau)\right\|_{L_{x}^{\infty}}+\left\|\nabla_{x} B(\tau)\right\|_{L_{x}^{\infty}} d \tau\right) .
$$

By the same way, $\partial_{x_{2}} B$ and $\nabla_{x} E$ terms are similarly estimated, and we obtain a Grönwall type inequality. The proof of the lemma is completed by Grönwall's lemma.

We then estimate $\nabla_{x, v} f(t)$, and obtain the following lemma.

Lemma 3.2: Let $(f, E, B)$ be a continuously differentiable solution of the VMCS system (1.2)-(1.7), where f has compact support for each $t$. Suppose that initial data are finite, i.e.,

$$
\left\|\nabla_{x, v}^{\alpha} f(0)\right\|_{L_{x, v}^{\infty}}+\left\|\nabla_{x}^{\beta} E(0)\right\|_{L_{x}^{\infty}}+\left\|\nabla_{x}^{\beta} B(0)\right\|_{L_{x}^{\infty}}<\infty \quad \text { for } \quad|\alpha| \leq 1 \text { and }|\beta| \leq 2,
$$

and there exists a nondecreasing function $C(t)$ such that

$$
f(t, x, v)=0 \quad \text { for } \quad|v| \geq C(t) .
$$

Then, we have the following estimations:

$$
\left\|\nabla_{t, x, v}^{\alpha} f(t)\right\|_{L_{x, v}^{\infty}}+\left\|\nabla_{t, x}^{\alpha} E(t)\right\|_{L_{x}^{\infty}}+\left\|\nabla_{t, x}^{\alpha} B(t)\right\|_{L_{x}^{\infty}} \leq C(t) \quad \text { for } \quad|\alpha| \leq 1 .
$$


Proof: Note that the Vlasov equation (1.2) and the time evolution equation for the magnetic field (1.6) are the same ones as in the VM case. Hence, $\partial_{t} f$ and $\partial_{t} B$ are similarly estimated. On the other hand, the time derivative of $E$ should be estimated as

$$
\left\|\partial_{t} E(t)\right\|_{L_{x}^{\infty}} \leq\left\|\nabla_{x} B(t)\right\|_{L_{x}^{\infty}}+\|E(t)\|_{L_{x}^{\infty}}+\|j(t)\|_{L_{x}^{\infty}} \leq C(t),
$$

where we used (1.4) and (1.5), and this completes the proof.

A well-known iteration scheme is applied to get an existence theorem under the initial conditions given in Lemma 3.2 with the assumption of existence of a nondecreasing function $C(t)$. The 0th iteration functions are defined as initial data. If the $(m-1)$ th iteration functions are given, then we get the $m$ th iteration function $f^{(m)}$ from the following linear Vlasov equation:

$$
\partial_{t} f^{(m)}+\hat{v} \cdot \nabla_{x} f^{(m)}+F^{(m-1)} \cdot \nabla_{v} f^{(m)}=0 .
$$

From $f^{(m)}$, we obtain $\rho^{(m)}$ and $j^{(m)}$ by (1.7), and again from $\rho^{(m)}$ and $j^{(m)}$, we obtain $E^{(m)}$ and $B^{(m)}$ by solving linear inhomogeneous Klein-Gordon equations:

$$
\begin{aligned}
& \partial_{t}^{2} E^{(m)}-\Delta E^{(m)}+E^{(m)}=-\nabla_{x} \rho^{(m)}-\partial_{t} j^{(m)}+\left(j_{2}^{(m)},-j_{1}^{(m)}\right), \\
& \partial_{t}^{2} B^{(m)}-\Delta B^{(m)}+B^{(m)}=\partial_{x_{1}} j_{2}^{(m)}-\partial_{x_{2}} j_{1}^{(m)}-\rho^{(m)},
\end{aligned}
$$

and this completes the iteration scheme. Lemma 3.2 is well applied to each iteration step, and its convergence to a continuously differentiable function is then proved (see Ref. 8 for details). We obtain the following conditional existence theorem.

Proposition 3.1: Suppose that initial data of the VMCS system satisfy the following conditions:

1. $f(0)=f(0, x, v)$ is nonnegative, compactly supported, and $C^{1}$.

2. $E(0)=E(0, x)$ and $B(0)=B(0, x)$ are $C^{2}$ and satisfy

$$
\partial_{x_{1}} E_{1}(0)+\partial_{x_{2}} E_{2}(0)-B(0)=4 \pi \int f(0, v) d v .
$$

3. The following quantities are bounded:

$$
\left\|\nabla_{x, v}^{\alpha} f(0)\right\|_{L_{x, v}^{\infty}}+\left\|\nabla_{x}^{\beta} E(0)\right\|_{L_{x}^{\infty}}+\left\|\nabla_{x}^{\beta} B(0)\right\|_{L_{x}^{\infty}}<\infty \quad \text { for } \quad|\alpha| \leq 1 \quad \text { and } \quad|\beta| \leq 2 .
$$

If there exists a nondecreasing function $C(t)$ such that

$$
f(t, x, v)=0 \quad \text { for } \quad|v| \geq C(t),
$$

then there exists a unique $C^{1}$ solution to the VMCS system.

\section{A. Global existence}

Proposition 3.1 shows that a solution exists when a nondecreasing function $C(t)$ satisfying (3.1) exists. In this part, we show that such a nondecreasing function exists on $[0, \infty)$, hence global existence is obtained. We first state a lemma for the energy identity.

Lemma 3.3: Let $(f, E, B)$ be the solution given in Proposition 3.1. Then the following energy identity holds:

$$
\partial_{t}\left(4 \pi \int \sqrt{1+|v|^{2}} f d v+\frac{1}{2}|E|^{2}+\frac{1}{2} B^{2}\right)+\nabla_{x} \cdot\left(4 \pi \int v f d v+B\left(E_{2},-E_{1}\right)\right)=0 .
$$

Proof. We first multiply $\sqrt{1+|v|^{2}}$ to the Vlasov equation (1.2),

$$
\begin{aligned}
& \sqrt{1+|v|^{2}} \times\left(\partial_{t} f+\hat{v} \cdot \nabla_{x} f+F \cdot \nabla_{v} f=0\right) \\
& \Longleftrightarrow \quad \partial_{t}\left[\sqrt{1+|v|^{2}} f\right]+\hat{v} \cdot \nabla_{x}\left[\sqrt{1+|v|^{2}} f\right]+F \cdot \nabla_{v}\left[\sqrt{1+|v|^{2}} f\right]-F \cdot \hat{v} f=0,
\end{aligned}
$$


and then integrate it over $v \in \mathbb{R}^{2}$,

$$
\partial_{t} \int \sqrt{1+|v|^{2}} f d v+\nabla_{x} \cdot \int v f d v-\int F \cdot \hat{v} f d v=0
$$

The last term is calculated as follows:

$$
\begin{aligned}
-4 \pi \int F \cdot \hat{v} f d v & =-4 \pi \int E \cdot \hat{v} f d v=-E \cdot j=-E_{1} j_{1}-E_{2} j_{2} \\
& =E_{1}\left(\partial_{t} E_{1}-\partial_{x_{2}} B+E_{2}\right)+E_{2}\left(\partial_{t} E_{2}+\partial_{x_{1}} B-E_{1}\right) \\
& =\frac{1}{2} \partial_{t}|E|^{2}-E_{1} \partial_{x_{2}} B+E_{2} \partial_{x_{1}} B \\
& =\frac{1}{2} \partial_{t}|E|^{2}+\nabla_{x} \cdot\left[\left(E_{2} B,-E_{1} B\right)\right]-B\left(\partial_{x_{1}} E_{2}-\partial_{x_{2}} E_{1}\right) \\
& =\frac{1}{2} \partial_{t}|E|^{2}+\frac{1}{2} \partial_{t} B^{2}+\nabla_{x} \cdot\left[\left(E_{2} B,-E_{1} B\right)\right] .
\end{aligned}
$$

Therefore, we obtain the energy identity:

$$
\partial_{t}\left(4 \pi \int \sqrt{1+|v|^{2}} f d v+\frac{1}{2}|E|^{2}+\frac{1}{2} B^{2}\right)+\nabla_{x} \cdot\left(4 \pi \int v f d v+B\left(E_{2},-E_{1}\right)\right)=0 .
$$

The above lemma shows that the energy identity is identical to the VM case. ${ }^{12}$ Under suitable initial conditions, the energy identity can be integrated over $x \in \mathbb{R}^{2}$ : since $\dot{x}=\hat{v}$ and $|\hat{v}|<1$, it is easy to see that $f(t, x, v)=0$ if $|x| \geq C+t$ for some constant $C$. Hence, we obtain a well-known identity for total energy:

$$
4 \pi \iint \sqrt{1+|v|^{2}} f d v d x+\frac{1}{2} \int|E|^{2}+B^{2} d x=\text { const } .
$$

The fact that the energy identities for the VMCS and the VM cases are same is basically due to the symmetry property of (1.1). In other words, the symmetry of the Maxwell case still holds in the Maxwell-Chern-Simons case. On the other hand, since each field component satisfies the KleinGordon equation, we can compare the above energy identity with that of the Vlasov-Klein-Gordon case. ${ }^{19}$ The energy identity for the Vlasov-Klein-Gordon system is as follows:

$$
\iint \sqrt{1+|v|^{2}} f d v d x+\frac{1}{2} \int\left|\partial_{t} u\right|^{2}+\left|\nabla_{x} u\right|^{2}+|u|^{2} d x+\int \rho u d x=\text { const } .
$$

where $u$ is the scalar Klein-Gordon field, which does not need to be nonnegative, hence which makes a difficulty in controlling the third integral above. This problem does not appear in the VMCS case due to its symmetry.

Since the energy identity for the VMCS system is identical to that for the VM system, all the arguments from the VM case, which are based on the energy identity, can be applied to the VMCS case. For instance, Lemma 1 of Ref. 13 still holds in the VMCS case. Hence, we follow the arguments with the same energy identity, and then Proposition 3.1 is extended to the following global existence theorem for the VMCS system.

Theorem 3.1: Let initial data $f(0), E(0)$, and $B(0)$ satisfy the following conditions:

1. $f(0)=f(0, x, v)$ is nonnegative, compactly supported, and $C^{1}$.

2. $E(0)=E(0, x)$ and $B(0)=B(0, x)$ are $C^{2}$ and satisfy

$$
\partial_{x_{1}} E_{1}(0)+\partial_{x_{2}} E_{2}(0)-B(0)=4 \pi \int f(0, v) d v .
$$

3. The following quantities are bounded:

$$
\left\|\nabla_{x, v}^{\alpha} f(0)\right\|_{L_{x, v}^{\infty}}+\left\|\nabla_{x}^{\beta} E(0)\right\|_{L_{x}^{\infty}}+\left\|\nabla_{x}^{\beta} B(0)\right\|_{L_{x}^{\infty}}<\infty \quad \text { for } \quad|\alpha| \leq 1 \text { and }|\beta| \leq 2 .
$$


Then, there exists a unique global $C^{1}$ solution to the VMCS system such that there exists a nondecreasing function $C(t)$ satisfying

$$
\begin{gathered}
f(t, x, v)=0 \quad \text { for } \quad|v| \geq C(t), \\
\left\|\nabla_{t, x, v}^{\alpha} f(t)\right\|_{L_{x, v}^{\infty}}+\left\|\nabla_{t, x}^{\alpha} E(t)\right\|_{L_{x}^{\infty}}+\left\|\nabla_{t, x}^{\alpha} B(t)\right\|_{L_{x}^{\infty}} \leq C(t) \quad \text { for } \quad|\alpha| \leq 1 .
\end{gathered}
$$

\section{NONRELATIVISTIC LIMIT OF THE VMCS SYSTEM}

In this section, we study nonrelativistic limit of the VMCS system. The speed of light $c$ is used as a parameter, and it is proved that solutions to the VMCS system converge to solutions to the Vlasov-Yukawa system as $c$ tends to infinity.

The VY system reads as follows in two dimensions:

$$
\begin{gathered}
\partial_{t} f^{\infty}+v \cdot \nabla_{x} f^{\infty}+E^{\infty} \cdot \nabla_{v} f^{\infty}=0, \\
E^{\infty}=\frac{1}{2 \pi} \int_{\mathbb{R}^{2}} K_{1}(|x-y|) \frac{(x-y)}{|x-y|} \rho^{\infty}(t, y) d y, \\
\rho^{\infty}(t, x)=4 \pi \int_{\mathbb{R}^{2}} f^{\infty}(t, x, v) d v,
\end{gathered}
$$

where $K_{1}$ is the Bessel function of the second kind of order one. Note that $E^{\infty}$ can be written as $E^{\infty}=\nabla_{x} \phi$, where $\phi$ is defined by

$$
\phi(t, x)=-\frac{1}{2 \pi} \int_{\mathbb{R}^{2}} K_{0}(|x-y|) \rho^{\infty}(t, y) d y,
$$

where we used the second formula of (1.20), and $\phi$ satisfies

$$
-\Delta \phi+\phi=-\rho^{\infty} \text {. }
$$

Global existence of classical solutions to the VY system (4.1)-(4.3) is easily proved by applying well known arguments for the Vlasov-Poisson case, for instance see Appendix A of Ref. 5.

On the other hand, we need global existence theorems for the VMCS system for arbitrary $c$ cases, but we obtain those existence theorems by scaling. When $(f, E, B)$ is the solution given in Theorem 3.1, we can find a solution $\left(f^{c}, E^{c}, B^{c}\right)$ of the VMCS system for arbitrary $c$ as follows:

$$
f(t, x, v)=f^{c}\left(c^{-1} t, x, c v\right), \quad E(t, x)=c^{-2} E^{c}\left(c^{-1} t, x\right), \quad B(t, x)=c^{-2} B^{c}\left(c^{-1} t, x\right) .
$$

The above scaling insures global existence for any $c$. Estimations of $\left(f^{c}, E^{c}, B^{c}\right)$ may depend on $c$, but we obtain the following estimations, Lemma 4.1, which guarantees a uniform boundedness with respect to $c$ on a finite time interval. Below, we omit the superscript $c$, and $(f, E, B)$ will denote solutions of the VMCS system for arbitrary $c$ cases.

Lemma 4.1: Let $(f, E, B)$ be the solution to the VMCS system given in Theorem 3.1 for arbitrary $c$ cases. Then, there exists $T>0$ independent of $c$ such that there exists a nondecreasing function $C(t)$ on $[0, T]$ such that the following estimations hold:

$$
\begin{gathered}
f(t, x, v)=0 \quad \text { for } \quad|v| \geq C(t), \\
\|E(t)\|_{L_{x}^{\infty}}+\|B(t)\|_{L_{x}^{\infty}}+\left\|\nabla_{t, x, v} f(t)\right\|_{L_{x, v}^{\infty} \leq C(t) .}
\end{gathered}
$$

Proof: This lemma is proved by the same arguments given in Ref. 21, hence we skip the proof. 
We first state the theorem, and its proof will be given in Subsections IV A-IV C.

Theorem 4.1: Let $(f, E, B)$ be a $C^{1}$ solution to the VMCS system (1.2)-(1.7) with the following initial data.

$$
f(0), \quad E(0)=\nabla_{x} B(0), \quad B(0, x)=-2 \iint_{\mathbb{R}^{2} \times \mathbb{R}^{2}} K_{0}(|x-y|) f(0, y, v) d v d y,
$$

where $f(0)$ is a $C^{2}$ function with compact support. Let $\left(f^{\infty}, E^{\infty}\right)$ be a $C^{1}$ solution to the VY system (4.1)-(4.3) with the same initial data $f^{\infty}(0)=f(0)$. Then, the following estimations hold:

$$
\begin{gathered}
\left|f(t, x, v)-f^{\infty}(t, x, v)\right| \leq D c^{-\frac{1}{2}} \quad \text { on } \quad[0, T], \\
\left|E(t, x)-E^{\infty}(t, x)\right|+|B(t, x)-\phi(t, x)|+\left|E(t, x)-\nabla_{x} B(t, x)\right| \leq D c^{-\frac{1}{2}} \quad \text { on } \quad\left[c^{-\frac{1}{2}}, T\right],
\end{gathered}
$$

for any $x \in \mathbb{R}^{2}$ and $v \in \mathbb{R}^{2}$. Here, $\phi$ is the quantity given by (4.4) satisfying $E^{\infty}=\nabla_{x} \phi$, and $T$ is the number given in Lemma 4.1.

Remark 4.1: The initial conditions on $E(0)$ and $B(0)$ are consistent with the constraint equation (1.3). Due to the first condition, $E(0)=\nabla_{x} B(0), E q$. (1.3) gives

$$
\Delta B-B=\rho,
$$

and the condition on $B(0)$ satisfies the above equation. Note that $\nabla_{x}^{\alpha} E(0)$ decays exponentially fast at infinity for $|\alpha| \leq 2$ by Lemma 1.3. This property will be used in Lemma 4.4.

In Ref. 21, a stronger result was obtained. Solutions of the VM system converge to the VlasovPoisson system uniformly on [0, T], while the estimations for the field components in Theorem 4.1 hold on $\left[c^{-\frac{1}{2}}, T\right]$. This seems to be a technical problem in controlling $\tilde{E}^{F}$ and $\tilde{B}^{F}$. However, the result on the magnetic field $B$ is new. The B field was shown to vanish in the nonrelativistic limit in the VM case in Ref. 21. In the VMCS case, it is proved that the B field does not vanish but converges to a Yukawa potential $\phi$. Moreover, it is interesting that the electric field $E$ converges to $\nabla_{x} B$.

\section{A. Calculations for $E$ and $B$}

In this part, we study asymptotic behavior of $E$ and $B$ as the speed of light $c$ tends to infinity. Below, $T$ denotes the number given in Lemma 4.1 and $t_{0}$ a small number such that

$$
0<t_{0}<\min \{1, T\},
$$

which will be determined later.

Lemma 4.2: Consider the representations of $E$ and B given in Lemma 2.1. Then, we have the following estimations:

$$
E_{k}^{S}=B^{S}=B^{T}=\tilde{E}_{1}^{T_{2}}=\tilde{E}_{2}^{T_{1}}=\tilde{B}^{T_{k}}=O\left(c^{-1}\right) \quad \text { on } \quad[0, T] \quad \text { for } \quad k=1,2 .
$$

Proof: The above estimations are proved by the same calculations given in Ref. 21, hence we skip the proof.

Lemma 4.3: Consider the representations of $E$ and B given in Lemma 2.1. Then, we have the following estimations:

$$
\tilde{E}_{k}^{T_{k}}=O\left(\left(c t_{0}\right)^{-1}\right) \quad \text { on } \quad\left[t_{0}, T\right] \quad \text { for } \quad k=1,2 .
$$

Proof: We first follow the calculations of Ref. 21 and obtain the following estimation:

$$
\tilde{E}_{k}^{T_{k}}=\iint \frac{-2 \cos \sqrt{c^{2} t^{2}-|x-y|^{2}}\left(y_{k}-x_{k}\right)}{c t \sqrt{c^{2} t^{2}-|x-y|^{2}}} f(0, y, v) d v d y+O\left(c^{-1}\right) \quad \text { on } \quad[0, T],
$$


where the integration domain is $\left\{(y, v) \in \mathbb{R}^{2} \times \mathbb{R}^{2}:|x-y| \leq c t\right\}$. It is enough to show that the integral above is $O\left(\left(c t_{0}\right)^{-1}\right)$ on $\left[t_{0}, T\right]$. For $k=1$, we proceed the estimation as follows:

$$
\begin{aligned}
& \iint_{|x-y| \leq c t} \frac{-2 \cos \sqrt{c^{2} t^{2}-|x-y|^{2}}}{c t \sqrt{c^{2} t^{2}-|x-y|^{2}}}\left(y_{1}-x_{1}\right) f(0, y, v) d v d y \\
& \quad=-\frac{2}{c t} \int_{0}^{c t} \iint_{|\omega|=1} \frac{\cos \sqrt{c^{2} t^{2}-r^{2}}}{\sqrt{c^{2} t^{2}-r^{2}}} r \omega_{1} f(0, x+r \omega, v) r d v d \omega d r \\
& \quad=\frac{2}{c t} \int_{0}^{c t} \iint_{|\omega|=1} \partial_{r}\left[\sin \sqrt{c^{2} t^{2}-r^{2}}\right] \omega_{1} f(0, x+r \omega, v) r d v d \omega d r
\end{aligned}
$$

and we take integration by parts.

$$
\begin{aligned}
\frac{2}{c t} \int_{0}^{c t} \iint_{|\omega|=1} \partial_{r}\left[\sin \sqrt{c^{2} t^{2}-r^{2}}\right] \omega_{1} f(0, x+r \omega, v) r d v d \omega d r \\
=-\frac{2}{c t} \int_{0}^{c t} \iint_{|\omega|=1} \sin \sqrt{c^{2} t^{2}-r^{2}} \omega_{1} \omega \cdot \nabla_{x} f(0, x+r \omega, v) r d v d \omega d r \\
\quad-\frac{2}{c t} \int_{0}^{c t} \iint_{|\omega|=1} \sin \sqrt{c^{2} t^{2}-r^{2}} \omega_{1} f(0, x+r \omega, v) d v d \omega d r \\
=-\frac{2}{c t} \iint_{|x-y| \leq c t} \sin \sqrt{c^{2} t^{2}-|x-y|^{2}} \frac{\left(y_{1}-x_{1}\right)}{|x-y|} \frac{(y-x)}{|x-y|} \cdot \nabla_{x} f(0, y, v) d v d y \\
\quad-\frac{2}{c t} \iint_{|x-y| \leq c t} \sin \sqrt{c^{2} t^{2}-|x-y|^{2}} \frac{\left(y_{1}-x_{1}\right)}{|x-y|} f(0, y, v) \frac{1}{|x-y|} d v d y .
\end{aligned}
$$

We denote the first and the second integrals by $I_{1}$ and $I_{2}$, respectively. They are estimated as follows:

$$
\begin{aligned}
\left|I_{1}\right| & \leq D \frac{1}{c t} \iint_{|x-y| \leq c t}\left|\nabla_{x} f(0, y, v)\right| d v d y \\
& \leq D \frac{1}{c t} \iint_{\mathbb{R}^{2} \times \mathbb{R}^{2}} \chi_{\{|y| \leq R,|v| \leq R\}} d v d y \\
& \leq D \frac{1}{c t} \\
& \leq D \frac{1}{c t_{0}}
\end{aligned}
$$

where we used $t \geq t_{0}$ in the last inequality. Similarly, we have the following estimation:

$$
\begin{aligned}
\left|I_{2}\right| & \leq D \frac{1}{c t} \iint_{|x-y| \leq c t}|f(0, y, v)| \frac{1}{|x-y|} d v d y \\
& \leq D \frac{1}{c t_{0}} \int_{|y| \leq R} \int_{|v| \leq R} \frac{1}{|x-y|} d v d y \\
& \leq D \frac{1}{c t_{0}} .
\end{aligned}
$$

Hence, both $I_{1}$ and $I_{2}$ are $O\left(\left(c t_{0}\right)^{-1}\right)$ on $\left[t_{0}, T\right]$. The calculation for $\tilde{E}_{2}^{T_{2}}$ is exactly same, and this completes the proof.

Lemma 4.4: Consider the representations of $E$ and $B$ given in Lemma 2.1. Then, we have the following estimations:

$$
\tilde{E}_{k}^{F}=\tilde{B}^{F}=O\left(\left(c t_{0}\right)^{-1}\right) \quad \text { on } \quad\left[t_{0}, T\right] \quad \text { for } \quad k=1,2 .
$$


Proof: Initial conditions on $\partial_{t} E$ and $\partial_{t} B$ are determined by $E(0), B(0)$, and $f(0)$ through Eqs. (1.4)-(1.6). Due to the additional condition on $E(0)$ and $B(0)$, i.e., $E(0)=\nabla_{x} B(0)$, given in Theorem 4.1, initial conditions on $\partial_{t} E$ and $\partial_{t} B$ are given as follows:

$$
\partial_{t} E(0)=-j, \quad \partial_{t} B(0)=0 .
$$

We apply the above conditions to $\tilde{E}_{k}^{F}$ and $\tilde{B}^{F}$ as follows:

$$
\begin{gathered}
\tilde{E}_{k}^{F}=\frac{1}{2 \pi c}\left(\int \frac{\cos \sqrt{c^{2} t^{2}-|x-y|^{2}}}{\sqrt{c^{2} t^{2}-|x-y|^{2}}}\left(-j_{k}\right)(0, y) d y+\frac{\partial}{\partial t}\left[\int \frac{\cos \sqrt{c^{2} t^{2}-|x-y|^{2}}}{\sqrt{c^{2} t^{2}-|x-y|^{2}}} E_{k}(0, y) d y\right]\right), \\
\tilde{B}^{F}=\frac{1}{2 \pi c} \frac{\partial}{\partial t}\left[\int \frac{\cos \sqrt{c^{2} t^{2}-|x-y|^{2}}}{\sqrt{c^{2} t^{2}-|x-y|^{2}}} B(0, y) d y\right],
\end{gathered}
$$

where the integration domains are $\{y:|x-y| \leq c t\}$. The first integral of $\tilde{E}_{k}^{F}$ above is $O\left(c^{-1}\right)$ on $[0, T]$ by the same calculations in Ref. 21 . For simplicity, let $G=G(x)$ denote $E_{k}(0, x)$ or $B(0, x)$, and consider the following quantity:

$$
\frac{\partial}{\partial t}\left[\int_{|x-y| \leq c t} \frac{\cos \sqrt{c^{2} t^{2}-|x-y|^{2}}}{\sqrt{c^{2} t^{2}-|x-y|^{2}}} G(y) d y\right] .
$$

Then, it suffices to show that the above quantity is bounded uniformly on $c$

We first apply the additional condition, $E(0)=\nabla_{x} B(0)$, to the constraint equation (1.3) to obtain the following equations:

$$
\Delta B(0)-B(0)=\rho(0) .
$$

Since $f(0)$ is $C^{2}$, so is $\rho(0)$, we can apply Lemma 1.3. Consequently, $\nabla_{x}^{\alpha} E(0, x)$ and $\nabla_{x}^{\beta} B(0, x)$ have the exponentially decaying property for $|\alpha| \leq 2$ and $|\beta| \leq 3$, i.e., bounded by $D e^{-\frac{1}{2}|x|}$ for some positive constant $D$. Hence, we obtain

$$
\left|\nabla_{x}^{\alpha} G(x)\right| \leq D e^{-\frac{1}{2}|x|} \text { for }|\alpha| \leq 2 .
$$

We proceed the calculation of (4.5) as follows. By a change of variables,

$$
\frac{\partial}{\partial t} \int_{|x-y| \leq c t} \frac{\cos \sqrt{c^{2} t^{2}-|x-y|^{2}}}{\sqrt{c^{2} t^{2}-|x-y|^{2}}} G(y) d y=\frac{\partial}{\partial t} \int_{|\omega| \leq 1} \frac{\cos \left(c t \sqrt{1-|\omega|^{2}}\right)}{\sqrt{1-|\omega|^{2}}} G(x+c t \omega) c t d \omega,
$$

and then we can take the differentiation directly. Consequently, we obtain

$$
\begin{aligned}
& -\frac{1}{t} \int_{|x-y| \leq c t} \sin \sqrt{c^{2} t^{2}-|x-y|^{2}} G(y) d y+\frac{1}{t} \int_{|x-y| \leq c t} \frac{\cos \sqrt{c^{2} t^{2}-|x-y|^{2}}}{\sqrt{c^{2} t^{2}-|x-y|^{2}}} G(y) d y \\
& +\frac{1}{t} \int_{|x-y| \leq c t} \frac{\cos \sqrt{c^{2} t^{2}-|x-y|^{2}}}{\sqrt{c^{2} t^{2}-|x-y|^{2}}}(y-x) \cdot \nabla_{x} G(y) d y,
\end{aligned}
$$

and denote them by $I_{1}+I_{2}+I_{3}$, respectively. $I_{1}$ is easily estimated as follows:

$$
\left|I_{1}\right| \leq D \frac{1}{t} \int_{\mathbb{R}^{2}} e^{-\frac{1}{2}|y|} d y \leq D \frac{1}{t_{0}},
$$

where we used (4.6). Estimations for $I_{2}$ and $I_{3}$ are almost same. We only consider $I_{3}$ term. By a change of variables,

$$
\begin{aligned}
I_{3} & =\frac{1}{t} \int_{0}^{c t} \int_{|\omega|=1} \frac{\cos \sqrt{c^{2} t^{2}-r^{2}}}{\sqrt{c^{2} t^{2}-r^{2}}} r^{2} \omega \cdot \nabla_{x} G(y) d \omega d r \\
& =\frac{1}{t} \int_{0}^{c t} \int_{|\omega|=1} \partial_{r}\left[-\sin \sqrt{c^{2} t^{2}-r^{2}}\right] r \omega \cdot \nabla_{x} G(x+r \omega) d \omega d r .
\end{aligned}
$$


We now take integration by parts with respect to $r$. Then, boundary terms, $r=c t$ and $r=0$, vanish, and we have the following quantities:

$$
\begin{aligned}
I_{3}= & \frac{1}{t} \int_{0}^{c t} \int_{|\omega|=1} \sin \sqrt{c^{2} t^{2}-r^{2}} \omega \cdot \nabla_{x} G(x+r \omega) d \omega d r \\
& +\frac{1}{t} \int_{0}^{c t} \int_{|\omega|=1} \sin \sqrt{c^{2} t^{2}-r^{2}} r \sum_{i, j} \omega_{i} \omega_{j} \partial_{i} \partial_{j} G(x+r \omega) d \omega d r \\
= & \frac{1}{t} \int_{|x-y| \leq c t} \sin \sqrt{c^{2} t^{2}-|x-y|^{2}} \omega \cdot \nabla_{x} G(y) \frac{1}{r} d y \\
& +\frac{1}{t} \int_{|x-y| \leq c t} \sin \sqrt{c^{2} t^{2}-|x-y|^{2}} \sum_{i, j} \omega_{i} \omega_{j} \partial_{i} \partial_{j} G(y) d y .
\end{aligned}
$$

It is easy to see that by (4.6),

$$
\left|I_{3}\right| \leq D \frac{1}{t} \int_{\mathbb{R}^{2}} \frac{1}{r} e^{-\frac{1}{2}|y|} d y+D \frac{1}{t} \int_{\mathbb{R}^{2}} e^{-\frac{1}{2}|y|} d y \leq D \frac{1}{t_{0}} .
$$

Together with a similar estimation of $I_{2}$, it is shown that the quantity (4.5) is uniformly bounded with respect to $c$ on $\left[t_{0}, T\right]$. This completes the proof.

Lemma 4.5: Consider the representations of $E$ and B given in Lemma 2.1. Then, we have the following estimations:

$$
\begin{gathered}
E_{k}^{T}(t, x)=2 \iint_{\mathbb{R}^{2} \times \mathbb{R}^{2}} K_{1}(|x-y|) \frac{\left(x_{k}-y_{k}\right)}{|x-y|} f(t, y, v) d v d y+O\left(\left(c t_{0}\right)^{-1}\right) \\
\text { on }\left[t_{0}, T\right] \quad \text { for } \quad k=1,2 .
\end{gathered}
$$

Proof: We first follow the calculations of Ref. 21 and obtain the following estimation:

$E_{k}^{T}(t, x)=-2 \iiint \frac{\cos \sqrt{c^{2}(t-\tau)^{2}-|x-y|^{2}}}{(t-\tau) \sqrt{c^{2}(t-\tau)^{2}-|x-y|^{2}}} \xi_{k} f(t, y, v) d v d y d \tau+O\left(c^{-1}\right) \quad$ on $\quad[0, T]$,

where the integration domain is $\{(\tau, y, v):|x-y| \leq c(t-\tau)\}$. The above integral is rewritten as follows:

$$
\begin{aligned}
& -2 \iiint \frac{\cos \sqrt{c^{2}(t-\tau)^{2}-|x-y|^{2}}}{(t-\tau) \sqrt{c^{2}(t-\tau)^{2}-|x-y|^{2}}} \xi_{k} f(t, y, v) d v d y d \tau \\
& \quad=-2 \int_{0}^{t} \int_{0}^{c(t-\tau)} \iint_{|\omega|=1} \frac{\cos \sqrt{c^{2}(t-\tau)^{2}-r^{2}}}{(t-\tau) \sqrt{c^{2}(t-\tau)^{2}-r^{2}}} \frac{r \omega_{k}}{c(t-\tau)} f(t, x+r \omega, v) r d v d \omega d r d \tau \\
& =-2 \int_{0}^{c t} \int_{0}^{t-\frac{r}{c}} \iint_{|\omega|=1} \frac{\cos \sqrt{c^{2}(t-\tau)^{2}-r^{2}}}{(t-\tau)^{2} \sqrt{c^{2}(t-\tau)^{2}-r^{2}}} \frac{r^{2} \omega_{k}}{c} f(t, x+r \omega, v) d v d \omega d \tau d r
\end{aligned}
$$

Let us consider $\tau$ integration above for each $r$. It is rewritten as follows:

$$
\frac{r^{2}}{c} \int_{0}^{t-\frac{r}{c}} \frac{\cos \sqrt{c^{2}(t-\tau)^{2}-r^{2}}}{(t-\tau)^{2} \sqrt{c^{2}(t-\tau)^{2}-r^{2}}} d \tau=\int_{0}^{\sqrt{\frac{c^{2} t^{2}}{r^{2}}-1}} \frac{\cos (r u)}{\left(1+u^{2}\right)^{3 / 2}} d u,
$$

where we used change of variables $\tau \leftrightarrow u$ defined as follows:

$$
\sqrt{\frac{c^{2}(t-\tau)^{2}}{r^{2}}-1}=u, \quad(t-\tau)^{2}=\frac{r^{2}}{c^{2}}\left(1+u^{2}\right), \quad d \tau=\frac{r u}{c \sqrt{1+u^{2}}} d u .
$$


We now use Basset's integral (1.19):

$$
\frac{r^{2}}{c} \int_{0}^{t-\frac{r}{c}} \frac{\cos \sqrt{c^{2}(t-\tau)^{2}-r^{2}}}{(t-\tau)^{2} \sqrt{c^{2}(t-\tau)^{2}-r^{2}}} d \tau=r K_{1}(r)-\int_{\sqrt{\frac{c^{2} t^{2}}{r^{2}}-1}}^{\infty} \frac{\cos (r u)}{\left(1+u^{2}\right)^{3 / 2}} d u,
$$

where $K_{1}$ is the modified Bessel function of the second kind. We apply the above formula to (4.8) to obtain the followings:

$$
\begin{aligned}
& -2 \iiint \frac{\cos \sqrt{c^{2}(t-\tau)^{2}-|x-y|^{2}}}{(t-\tau) \sqrt{c^{2}(t-\tau)^{2}-|x-y|^{2}}} \xi_{k} f(t, y, v) d v d y d \tau \\
& =-2 \int_{0}^{c t} \iint_{|\omega|=1} r K_{1}(r) \omega_{k} f(t, x+r \omega, v) d v d \omega d r \\
& \quad+2 \int_{0}^{c t} \int_{\sqrt{\frac{c^{2} t^{2}}{r^{2}}-1}}^{\infty} \iint_{|\omega|=1} \frac{\cos (r u)}{\left(1+u^{2}\right)^{3 / 2}} \omega_{k} f(t, x+r \omega, v) d v d \omega d u d r \\
& =2 \iint_{|x-y| \leq c t} K_{1}(|x-y|) \frac{\left(x_{k}-y_{k}\right)}{|x-y|} f(t, y, v) d v d y \\
& \quad+2 \iint_{|x-y| \leq c t} \int_{\sqrt{\frac{c^{2} t^{2}}{|x-y|^{2}}-1}}^{\infty} \frac{\cos (|x-y| u)}{\left(1+u^{2}\right)^{3 / 2}} \frac{\left(y_{k}-x_{k}\right)}{|x-y|^{2}} f(t, y, v) d u d v d y .
\end{aligned}
$$

Therefore, it suffices to show that the second integral above is $O\left(\left(c t_{0}\right)^{-1}\right)$ on $\left[t_{0}, T\right]$. Let us consider $u$ integration in the second integral. We use the change of variables (4.9) again to obtain $\tau$ integration. Below, $r$ denotes $|x-y|$ for simplicity:

$$
\begin{aligned}
& 2 \iint_{|x-y| \leq c t} \int_{\sqrt{\frac{c^{2} t^{2}}{r^{2}}-1}}^{\infty} \frac{\cos (r u)}{\left(1+u^{2}\right)^{3 / 2}} \frac{\left(y_{k}-x_{k}\right)}{|x-y|^{2}} f(t, y, v) d u d v d y \\
& \quad=2 \iint_{|x-y| \leq c t} \int_{-\infty}^{0} \frac{\cos \sqrt{c^{2}(t-\tau)^{2}-r^{2}}}{c(t-\tau)^{2} \sqrt{c^{2}(t-\tau)^{2}-r^{2}}}\left(y_{k}-x_{k}\right) f(t, y, v) d \tau d v d y .
\end{aligned}
$$

For each $r \leq c t$, the above $\tau$ integration is written as follows:

$$
\int_{-\infty}^{0} \frac{\cos \sqrt{c^{2}(t-\tau)^{2}-r^{2}}}{c(t-\tau)^{2} \sqrt{c^{2}(t-\tau)^{2}-r^{2}}} d \tau=\int_{c t}^{\infty} \frac{\cos \sqrt{s^{2}-r^{2}}}{s^{2} \sqrt{s^{2}-r^{2}}} d s .
$$

We estimate the last integration as follows:

$$
\begin{aligned}
\left|\int_{c t}^{2 c t} \frac{\cos \sqrt{s^{2}-r^{2}}}{s^{2} \sqrt{s^{2}-r^{2}}} d s\right| & \leq \int_{c t}^{2 c t} \frac{1}{s^{2} \sqrt{s+r} \sqrt{s-r}} d s \\
& \leq \frac{1}{(c t)^{5 / 2}} \int_{c t}^{2 c t} \frac{1}{\sqrt{s-r}} d s \\
& \leq D \frac{1}{(c t)^{2}}
\end{aligned}
$$

and

$$
\begin{aligned}
\left|\int_{2 c t}^{\infty} \frac{\cos \sqrt{s^{2}-r^{2}}}{s^{2} \sqrt{s^{2}-r^{2}}} d s\right| & \leq \int_{2 c t}^{\infty} \frac{1}{s^{2} \sqrt{s+r} \sqrt{s-r}} d s \\
& \leq D \frac{1}{(c t)^{3 / 2}} \int_{2 c t}^{\infty} \frac{1}{(s-r)^{3 / 2}} d s \\
& \leq D \frac{1}{(c t)^{2}}
\end{aligned}
$$


where we used $r \leq c t$ at the last inequality. We plug the above estimation into (4.10) and obtain the following estimation:

$$
\begin{aligned}
& \left|2 \iint_{|x-y| \leq c t} \int_{-\infty}^{0} \frac{\cos \sqrt{c^{2}(t-\tau)^{2}-r^{2}}}{c(t-\tau)^{2} \sqrt{c^{2}(t-\tau)^{2}-r^{2}}}\left(y_{k}-x_{k}\right) f(t, y, v) d \tau d v d y\right| \\
& \quad \leq D \iint_{|x-y| \leq c t} \frac{1}{(c t)^{2}}|x-y| f(t, y, v) d v d y \\
& \quad \leq D \frac{1}{c t} \iint_{\mathbb{R}^{2} \times \mathbb{R}^{2}} \chi_{\{|y| \leq R+t C(t),|v| \leq C(t)\}} d v d y \\
& \quad \leq D \frac{1}{c t_{0}}
\end{aligned}
$$

for some constant $D$. This completes the proof.

Lemma 4.6: Consider the representations of $E$ and B given in Lemma 2.1. Then, we have the following estimations:

$$
B^{N}(t, x)=-2 \iint_{\mathbb{R}^{2} \times \mathbb{R}^{2}} K_{0}(|x-y|) f(t, y, v) d v d y+O\left(\left(c t_{0}\right)^{-1}\right)+O\left(c^{-1} \log \left(c / t_{0}\right)\right) \quad \text { on } \quad\left[t_{0}, T\right] .
$$

Proof: We first rewrite $B^{N}$ as follows:

$$
B^{N}=\iint_{|x-y| \leq c t} \int_{0}^{t-\frac{r}{c}} \frac{\cos \sqrt{c^{2}(t-\tau)^{2}-r^{2}}}{\sqrt{c^{2}(t-\tau)^{2}-r^{2}}}(-2 c f)(\tau, y, v) d \tau d v d y,
$$

and apply the change of variables defined by (4.9):

$$
\begin{aligned}
B^{N} & =\iint_{|x-y| \leq c t} \int_{0}^{\sqrt{\frac{c^{2} t^{2}}{r^{2}}-1}} \frac{\cos (r u)}{\sqrt{1+u^{2}}}(-2 f)\left(t-\frac{r}{c} \sqrt{1+u^{2}}, y, v\right) d u d v d y \\
& =\int_{0}^{c t} \iint_{|\omega|=1} \int_{0}^{\sqrt{\frac{c^{2} t^{2}}{r^{2}}}-1} \frac{\cos (r u)}{\sqrt{1+u^{2}}}(-2 f)\left(t-\frac{r}{c} \sqrt{1+u^{2}}, x+r \omega, v\right) r d u d v d \omega d r \\
& =\int_{0}^{c t} \iint_{|\omega|=1} \int_{0}^{\sqrt{\frac{c^{2} t^{2}}{r^{2}}-1}} \frac{\partial_{u}[\sin (r u)]}{\sqrt{1+u^{2}}}(-2 f)\left(t-\frac{r}{c} \sqrt{1+u^{2}}, x+r \omega, v\right) d u d v d \omega d r .
\end{aligned}
$$

Let us consider the integrand above to take integration by parts with respect to $u$ variable. The integrand can be written as follows:

$$
\begin{aligned}
\partial_{u}[ & \left.\frac{\sin (r u)}{\sqrt{1+u^{2}}}(-2 f)\left(t-\frac{r}{c} \sqrt{1+u^{2}}, x+r \omega, v\right)\right] \\
& -\sin (r u) \partial_{u}\left[\frac{1}{\sqrt{1+u^{2}}}\right](-2 f)\left(t-\frac{r}{c} \sqrt{1+u^{2}}, x+r \omega, v\right) \\
& -\sin (r u) \frac{1}{\sqrt{1+u^{2}}}(-2) \partial_{u}\left[f\left(t-\frac{r}{c} \sqrt{1+u^{2}}, x+r \omega, v\right)\right],
\end{aligned}
$$

and we denote them by $I_{1}, I_{2}$, and $I_{3}$, respectively. $I_{1}$ and $I_{3}$ terms are easily estimated as follows:

$$
\begin{aligned}
\int_{0}^{c t} & \iint_{|\omega|=1} \int_{0}^{\sqrt{\frac{c^{2} t^{2}}{r^{2}}-1}} I_{1} d u d v d \omega d r \\
& =\int_{0}^{c t} \iint_{|\omega|=1} \frac{\sin \sqrt{c^{2} t^{2}-r^{2}}}{c t} r(-2 f)(0, x+r \omega, v) d v d \omega d r \\
& =\frac{1}{c t} \iint_{|x-y| \leq c t} \sin \sqrt{c^{2} t^{2}-r^{2}}(-2 f)(0, y, v) d v d y .
\end{aligned}
$$


Since the sine function is bounded and $f(0)$ has compact support, the last quantity is $O\left(\left(c t_{0}\right)^{-1}\right)$ on $\left[t_{0}, T\right]$. Hence, we obtain an estimation for $I_{1}$ term:

$$
\int_{0}^{c t} \iint_{|\omega|=1} \int_{0}^{\sqrt{\frac{c^{2} t^{2}}{r^{2}}-1}} I_{1} d u d v d \omega d r=O\left(\left(c t_{0}\right)^{-1}\right) \quad \text { on } \quad\left[t_{0}, T\right] .
$$

$I_{3}$ term is explicitly written as follows:

$$
I_{3}=-2 \frac{\sin (r u)}{\sqrt{1+u^{2}}} \frac{r}{c} \frac{u}{\sqrt{1+u^{2}}}\left(\partial_{t} f\right)\left(t-\frac{r}{c} \sqrt{1+u^{2}}, x+r \omega, v\right),
$$

and it is estimated as follows:

$$
\begin{aligned}
\mid \int_{0}^{c t} & \iint_{|\omega|=1} \int_{0}^{\sqrt{\frac{c^{2} t^{2}}{r^{2}}-1}}-2 \sin (r u) \frac{r}{c} \frac{u}{1+u^{2}}\left(\partial_{t} f\right)\left(t-\frac{r}{c} \sqrt{1+u^{2}}, x+r \omega, v\right) d u d v d \omega d r \mid \\
& =\left|\iint_{|x-y| \leq c t} \int_{0}^{\sqrt{\frac{c^{2} t^{2}}{r^{2}}-1}}-2 \sin (r u) \frac{1}{c} \frac{u}{1+u^{2}}\left(\partial_{t} f\right)\left(t-\frac{r}{c} \sqrt{1+u^{2}}, y, v\right) d u d v d y\right| \\
& \leq D \frac{1}{c} \iint_{|x-y| \leq c t} \int_{0}^{\sqrt{\frac{c^{2} t^{2}}{r^{2}}-1}} \frac{u}{1+u^{2}} \chi_{\{|y| \leq R+t C(t),|v| \leq C(t)\}} d u d v d y \\
& \leq D \frac{1}{c} \int_{|y| \leq R+t C(t)} \int_{|v| \leq C(t)}\left[\log \sqrt{1+u^{2}}\right]_{u=0}^{\sqrt{\frac{c^{2} t^{2}}{r^{2}}-1}} d v d y \\
& \leq D \frac{1}{c} \int_{|y| \leq R+t C(t)} \log \left(\frac{c t}{r}\right) d y \\
& \leq D \frac{1}{c} \log c-D \frac{1}{c} \log t_{0}+D \frac{1}{c}
\end{aligned}
$$

where we used $t_{0}<1$. Hence, we obtain an estimation for $I_{3}$ term:

$$
\int_{0}^{c t} \iint_{|\omega|=1} \int_{0}^{\sqrt{\frac{c^{2} t^{2}}{r^{2}}-1}} I_{3} d u d v d \omega d r=O\left(c^{-1} \log \left(c / t_{0}\right)\right) .
$$

We finally estimate $I_{2}$ term. It is explicitly written as follows:

$$
I_{2}=-\sin (r u) \frac{u}{\left(1+u^{2}\right)^{3 / 2}}(2 f)\left(t-\frac{r}{c} \sqrt{1+u^{2}}, x+r \omega, v\right),
$$

and by the change of variables defined by (4.9) to get again $\tau$ integration:

$$
\begin{aligned}
\int_{0}^{c t} & \iint_{|\omega|=1} \int_{0}^{\sqrt{\frac{c^{2} t^{2}}{r^{2}}-1}} I_{2} d u d v d \omega d r \\
& =-\int_{0}^{c t} \iint_{|\omega|=1} \int_{0}^{t-\frac{r}{c}} \sin \sqrt{c^{2}(t-\tau)^{2}-r^{2}} \frac{r}{c(t-\tau)^{2}}(2 f)(\tau, x+r \omega, v) d \tau d v d \omega d r \\
& =-\iint_{|x-y| \leq c t} \int_{0}^{t-\frac{r}{c}} \sin \sqrt{c^{2}(t-\tau)^{2}-r^{2}} \frac{1}{c(t-\tau)^{2}}(2 f)(\tau, y, v) d \tau d v d y .
\end{aligned}
$$

The last integrand can be rewritten as $I_{21}+I_{22}$ defined by

$$
\begin{gathered}
I_{21}=\sin \sqrt{c^{2}(t-\tau)^{2}-r^{2}} \frac{2}{c(t-\tau)^{2}}(f(\tau, y, v)-f(t, y, v)), \\
I_{22}=\sin \sqrt{c^{2}(t-\tau)^{2}-r^{2}} \frac{1}{c(t-\tau)^{2}}(2 f)(t, y, v) .
\end{gathered}
$$


Note that $I_{21}$ is estimated as follows:

$$
\begin{aligned}
\left|I_{21}\right| & \left.\leq \frac{2}{c(t-\tau)}\left|\partial_{t} f(\sigma, y, v)\right| \quad \text { (for some } \tau \leq \sigma \leq t\right) \\
& \leq \frac{D}{c(t-\tau)} \chi_{\{|y| \leq R+t C(t),|v| \leq C(t)\}} .
\end{aligned}
$$

Hence, we obtain the following estimation:

$$
\begin{aligned}
& \left|\iint_{|x-y| \leq c t} \int_{0}^{t-\frac{r}{c}} I_{21} d \tau d v d y\right| \\
& \quad \leq D \frac{1}{c} \int_{|y| \leq R+t C(t)} \int_{|v| \leq C(t)}[-\log (t-\tau)]_{\tau=0}^{t-\frac{r}{c}} d v d y \\
& \quad \leq D \frac{1}{c} \int_{|y| \leq R+t C(t)} \int_{|v| \leq C(t)} \log \left(\frac{c t}{r}\right) d v d y \\
& \quad \leq D \frac{1}{c} \log \left(c / t_{0}\right) .
\end{aligned}
$$

In order to estimate $I_{22}$ term, we use again the change of variables defined by (4.9) to get an integration for $u$ variable. Let us consider $\tau$ integration for $I_{22}$ term:

$$
\int_{0}^{t-\frac{r}{c}} \sin \sqrt{c^{2}(t-\tau)^{2}-r^{2}} \frac{1}{c(t-\tau)^{2}} d \tau=\int_{0}^{\sqrt{\frac{c^{2} t^{2}}{r^{2}}-1}} \frac{\sin (r u) u}{r\left(1+u^{2}\right)^{3 / 2}} d u .
$$

We now use Lemma 1.2, and then the above quantity is written as follows:

$$
\int_{0}^{t-\frac{r}{c}} \sin \sqrt{c^{2}(t-\tau)^{2}-r^{2}} \frac{1}{c(t-\tau)^{2}} d \tau=K_{0}(r)-\int_{\sqrt{\frac{c^{2} t^{2}}{r^{2}}-1}}^{\infty} \frac{\sin (r u) u}{r\left(1+u^{2}\right)^{3 / 2}} d u .
$$

Together with (4.13), $I_{2}$ term is estimated as follows:

$$
\begin{aligned}
\int_{0}^{c t} & \iint_{|\omega|=1} \int_{0}^{\sqrt{\frac{c^{2} t^{2}}{r^{2}}-1}} I_{2} d u d v d \omega d r \\
= & -\iint_{|x-y| \leq c t} \int_{0}^{t-\frac{r}{c}} I_{22} d \tau d v d y+O\left(c^{-1} \log c\right) \\
= & -\iint_{|x-y| \leq c t} K_{0}(r)(2 f)(t, y, v) d v d y \\
& +\iint_{|x-y| \leq c t} \int_{\sqrt{\frac{c^{2} t^{2}}{r^{2}}-1}}^{\infty} \frac{\sin (r u) u}{r\left(1+u^{2}\right)^{3 / 2}}(2 f)(t, y, v) d u d v d y+O\left(c^{-1} \log \left(c / t_{0}\right)\right) .
\end{aligned}
$$

Therefore, it is enough to show that the second integral of the last quantity is $O\left(\left(c t_{0}\right)^{-1}\right)$ on $\left[t_{0}, T\right]$. We use again the change of variables defined by (4.9). Then, we have the following estimation:

$$
\left|\int_{\sqrt{\frac{c^{2} t^{2}}{r^{2}}-1}}^{\infty} \frac{\sin (r u) u}{r\left(1+u^{2}\right)^{3 / 2}} d u\right|=\left|\frac{1}{c} \int_{-\infty}^{0} \frac{\sin \sqrt{c^{2}(t-\tau)^{2}-r^{2}}}{(t-\tau)^{2}} d \tau\right| \leq \frac{1}{c} \int_{-\infty}^{0} \frac{1}{(t-\tau)^{2}} d \tau \leq \frac{1}{c t_{0}} .
$$

Since $f(t)$ is supported in $\{|y| \leq R+t C(t),|v| \leq C(t)\}$, it is easy to show that

$$
\iint_{|x-y| \leq c t} \int_{\sqrt{\frac{c^{2} t^{2}}{r^{2}}-1}}^{\infty} \frac{\sin (r u) u}{r\left(1+u^{2}\right)^{3 / 2}}(2 f)(t, y, v) d u d v d y=O\left(\left(c t_{0}\right)^{-1}\right)
$$

on $\left[t_{0}, T\right]$. We combine (4.11), (4.12), (4.14), and (4.15) to obtain the desired result. 
Lemma 4.7: Consider the representations of $E$ and $B$ given in Lemma 2.1. Then, we have the following estimations:

$$
E_{k}^{N}=O\left(\left(c t_{0}\right)^{-1}\right)+O\left(c^{-1} \log \left(c / t_{0}\right)\right) \quad \text { on } \quad\left[t_{0}, T\right] \quad \text { for } \quad k=1,2 .
$$

Proof: Comparing $E_{k}^{N}$ with $B^{N}$, we can see that $E_{k}^{N} \approx c^{-1} B^{N}$. Hence, the lemma is proved by following the proof of the previous lemma for $B^{N}$. We skip the proof.

\section{B. Calculations for $\nabla_{x} B$}

In order to estimate $\nabla_{x} B$, we take $x$ derivatives on $B$ given in Lemma 2.1, and then use the differential operators $S$ and $T$ with (2.1):

$$
\partial_{x_{k}} B=\partial_{x_{k}} \tilde{B}^{F}+\partial_{x_{k}} \tilde{B}^{T_{1}}+\partial_{x_{k}} \tilde{B}^{T_{2}}+\partial_{x_{k}} B^{S}+\partial_{x_{k}} B^{T}+\partial_{x_{k}} B^{N}, \quad k=1,2 .
$$

By the same arguments as in Sec. IV A, it is shown that the following quantity is $O\left(\left(c t_{0}\right)^{-1}\right)$ :

$$
\partial_{x_{k}} \tilde{B}^{F}+\partial_{x_{k}} \tilde{B}^{T_{1}}+\partial_{x_{k}} \tilde{B}^{T_{2}}+\partial_{x_{k}} B^{S}+\partial_{x_{k}} B^{T}=O\left(\left(c t_{0}\right)^{-1}\right) .
$$

Hence, we only consider $\partial_{x_{k}} B^{N}$ term. It has the following representation:

$$
\partial_{x_{k}} B^{N}=\tilde{B}_{k}^{N}+B_{k}^{N S}+B_{k}^{N T},
$$

where $\tilde{B}_{k}^{N}$ is a quantity depending only on initial data, and

$$
\begin{aligned}
B_{k}^{N S}= & \iiint \frac{\cos \sqrt{c^{2}(t-\tau)^{2}-|x-y|^{2}}}{c \sqrt{c^{2}(t-\tau)^{2}-|x-y|^{2}}}(F f)(\tau, y, v) \cdot \nabla_{v}\left[\frac{-2 c \xi_{k}}{1+c^{-1} \hat{v} \cdot \xi}\right] d v d y d \tau, \\
B_{1}^{N T}= & \iiint \frac{\cos \sqrt{c^{2}(t-\tau)^{2}-|x-y|^{2}}}{c^{2}(t-\tau) \sqrt{c^{2}(t-\tau)^{2}-|x-y|^{2}}}\left(\frac{2\left(-c\left(c \xi_{1}+\hat{v}_{1}\right)-\hat{v}_{2}\left(\hat{v}_{1} \xi_{2}-\hat{v}_{2} \xi_{1}\right)\right)}{\left(1+c^{-1} \hat{v} \cdot \xi\right)^{2}}\right) \\
& \times f(\tau, y, v) d v d y d \tau, \\
B_{2}^{N T}= & \iiint \frac{\cos \sqrt{c^{2}(t-\tau)^{2}-|x-y|^{2}}}{c^{2}(t-\tau) \sqrt{c^{2}(t-\tau)^{2}-|x-y|^{2}}}\left(\frac{2\left(-c\left(c \xi_{2}+\hat{v}_{2}\right)+\hat{v}_{1}\left(\hat{v}_{1} \xi_{2}-\hat{v}_{2} \xi_{1}\right)\right)}{\left(1+c^{-1} \hat{v} \cdot \xi\right)^{2}}\right) \\
& \times f(\tau, y, v) d v d y d \tau,
\end{aligned}
$$

where the integration domains are $\left\{(\tau, y, v) \in[0, t] \times \mathbb{R}^{2} \times \mathbb{R}^{2}:|x-y| \leq c(t-\tau)\right\}$. Similarly again, $\tilde{B}_{k}^{N}$ and $B_{k}^{N S}$ terms can be shown to be $O\left(\left(c t_{0}\right)^{-1}\right)$ on $\left[t_{0}, T\right]$. Moreover, the following quantities are $O\left(c^{-1}\right)$ on $[0, T]$ :

$$
\iiint \frac{\cos \sqrt{c^{2}(t-\tau)^{2}-|x-y|^{2}}}{c^{2}(t-\tau) \sqrt{c^{2}(t-\tau)^{2}-|x-y|^{2}}}\left(\frac{2\left(-c \hat{v}_{1}-\hat{v}_{2}\left(\hat{v}_{1} \xi_{2}-\hat{v}_{2} \xi_{1}\right)\right)}{\left(1+c^{-1} \hat{v} \cdot \xi\right)^{2}}\right) f(\tau, y, v) d v d y d \tau
$$

for $k=1$, and

$$
\iiint \frac{\cos \sqrt{c^{2}(t-\tau)^{2}-|x-y|^{2}}}{c^{2}(t-\tau) \sqrt{c^{2}(t-\tau)^{2}-|x-y|^{2}}}\left(\frac{2\left(-c \hat{v}_{2}+\hat{v}_{1}\left(\hat{v}_{1} \xi_{2}-\hat{v}_{2} \xi_{1}\right)\right)}{\left(1+c^{-1} \hat{v} \cdot \xi\right)^{2}}\right) f(\tau, y, v) d v d y d \tau
$$

for $k=2$, which are a part of $B_{k}^{N T}$ (see (66) at p. 271 of Ref. 21). Consequently, we only need to consider the following quantity:

$$
\iiint \frac{\cos \sqrt{c^{2}(t-\tau)^{2}-|x-y|^{2}}}{(t-\tau) \sqrt{c^{2}(t-\tau)^{2}-|x-y|^{2}}}\left(\frac{-2 \xi_{k}}{\left(1+c^{-1} \hat{v} \cdot \xi\right)^{2}}\right) f(\tau, y, v) d v d y d \tau
$$

Since the term $\left(1+c^{-1} \hat{v} \cdot \xi\right)^{-2}$ is bounded by $C(t)$ (see (17) at p. 256 of Ref. 21), the above quantity is exactly same with (4.7) for $E_{k}^{T}$, and therefore we obtain the following lemma thanks to Lemma 4.5. 
Lemma 4.8: Consider the representations of $E$ and B given in Lemma 2.1. Then, we have the following estimations:

$$
E_{k}^{T}=\partial_{x_{k}} B+O\left(\left(c t_{0}\right)^{-1}\right) \quad \text { on } \quad\left[t_{0}, T\right] \quad \text { for } \quad k=1,2 .
$$

Remark 4.2: By the estimations given in Sec. IV A, E is written as $E=E^{T}+O\left(\left(c t_{0}\right)^{-1}\right)$ on $\left[t_{0}, T\right]$. Hence, Lemma 4.8 shows that $E=\nabla_{x} B+O\left(\left(c t_{0}\right)^{-1}\right)$ on $\left[t_{0}, T\right]$.

\section{Proof of Theorem 4.1}

We are now ready to prove Theorem 4.1. With the estimations in Secs. IV A and IV B, we follow the arguments of Ref. 21. Since the remaining arguments for the proof are almost same, we only present a sketch of it. Combining all the estimates, we obtain the followings:

$$
\begin{aligned}
& E(t, x)=2 \iint_{\mathbb{R}^{2} \times \mathbb{R}^{2}} K_{1}(|x-y|) \frac{(x-y)}{|x-y|} f(t, y, v) d v d y+O\left(\left(c t_{0}\right)^{-1}\right)+O\left(c^{-1} \log \left(c / t_{0}\right)\right), \\
& B(t, x)=-2 \iint_{\mathbb{R}^{2} \times \mathbb{R}^{2}} K_{0}(|x-y|) f(t, y, v) d v d y+O\left(\left(c t_{0}\right)^{-1}\right)+O\left(c^{-1} \log \left(c / t_{0}\right)\right),
\end{aligned}
$$

and recall that $E^{\infty}$ and $\phi$ are given by (4.2) and (4.4) as follows:

$$
\begin{gathered}
E^{\infty}(t, x)=2 \iint_{\mathbb{R}^{2} \times \mathbb{R}^{2}} K_{1}(|x-y|) \frac{(x-y)}{|x-y|} f^{\infty}(t, y, v) d v d y, \\
\phi(t, x)=-2 \iint_{\mathbb{R}^{2} \times \mathbb{R}^{2}} K_{0}(|x-y|) f^{\infty}(t, y, v) d v d y .
\end{gathered}
$$

Define

$$
H(t)=\sup \left\{\left|f(s, x, v)-f^{\infty}(s, x, v)\right|: 0 \leq s \leq t,(x, v) \in \mathbb{R}^{2} \times \mathbb{R}^{2}\right\} .
$$

Subtracting (4.18) and (4.19) from (4.16) and (4.17), we obtain for $t_{0} \leq t \leq T$,

$$
\left\|E(t)-E^{\infty}(t)\right\|_{L_{x}^{\infty}}+\|B(t)-\phi(t)\|_{L_{x}^{\infty}} \leq D H(t)+D\left(c t_{0}\right)^{-1}+D c^{-1} \log \left(c / t_{0}\right) .
$$

To estimate the distribution functions, we subtract (4.1) from (1.2), and obtain the following two inequalities:

$$
\mid \frac{d}{d s}\left[f\left(s, X(s), V(s)-f^{\infty}(s, X(s), V(s))\right] \mid \leq D H(s)+D\left(c t_{0}\right)^{-1}+D c^{-1} \log \left(c / t_{0}\right),\right.
$$

and clearly

$$
\mid \frac{d}{d s}\left[f\left(s, X(s), V(s)-f^{\infty}(s, X(s), V(s))\right] \mid \leq D,\right.
$$

where $(X(s), V(s))$ is the characteristic curve defined by (1.15). We integrate (4.21) from $t_{0}$ to $t$, and obtain a Grönwall type inequality:

$$
H(t) \leq H\left(t_{0}\right)+D\left(c t_{0}\right)^{-1}+D c^{-1} \log \left(c / t_{0}\right)+D \int_{t_{0}}^{t} H(s) d s,
$$

which implies

$$
H(t) \leq D H\left(t_{0}\right)+D\left(c t_{0}\right)^{-1}+D c^{-1} \log \left(c / t_{0}\right) \quad \text { on } \quad\left[t_{0}, T\right] .
$$

On the other hand, we use $f(0)=f^{\infty}(0)$ to integrate (4.22) from 0 to $t$, and obtain $H(t) \leq D t$ on $[0$, $T]$, in particular $H\left(t_{0}\right) \leq D t_{0}$. Consequently, (4.23) implies that

$$
H(t) \leq D t_{0}+D\left(c t_{0}\right)^{-1}+D c^{-1} \log \left(c / t_{0}\right),
$$


which holds on $[0, T]$, since $H$ is nondecreasing. We now set $t_{0}$ as follows:

$$
t_{0}=c^{-\frac{1}{2}}
$$

Hence, we have

$$
H(t) \leq D c^{-\frac{1}{2}} \quad \text { on } \quad[0, T] .
$$

Applying the above result to (4.20), we obtain the desired estimations:

$$
\begin{gathered}
\left|f(t, x, v)-f^{\infty}(t, x, v)\right| \leq D c^{-\frac{1}{2}} \quad \text { on } \quad[0, T] \\
\left|E(t, x)-E^{\infty}(t, x)\right|+|B(t, x)-\phi(t, x)| \leq D c^{-\frac{1}{2}} \quad \text { on } \quad\left[c^{-\frac{1}{2}}, T\right],
\end{gathered}
$$

for any $x \in \mathbb{R}^{2}$ and $v \in \mathbb{R}^{2}$. The estimation of $E-\nabla_{x} B$ given in Lemma 4.8 and Remark 4.2 is written as follows:

$$
\left|E(t, x)-\nabla_{x} B(t, x)\right| \leq D c^{-\frac{1}{2}} \quad \text { on } \quad\left[c^{-\frac{1}{2}}, T\right] .
$$

Therefore, this completes the proof of Theorem 4.1.

\section{ACKNOWLEDGMENTS}

The first author was supported by Basic Science Research Program through the National Research Foundation of Korea (NRF) funded by the Ministry of Education, Science and Technology (2011-0015866).

${ }^{1}$ M. Abramowitz, and I. A. Stegun, Handbook of Mathematical Functions with Formulas, Graphs, and Mathematical Tables, edited by M. Abramowitz, and I. A. Stegun (Dover, New York, 1966).

${ }^{2}$ S. Caprino, C. Marchioro, and M. Pulvirenti, "On the two-dimensional Vlasov-Helmholtz equation with infinite mass," Commun. Partial Differ. Equ. 27(3-4), 791-808 (2002).

${ }^{3}$ D. Chae and M. Chae, "The global existence in the Cauchy problem of the Maxwell-Chern-Simons-Higgs system," J. Math. Phys. 43(11), 5470-5482 (2002).

${ }^{4}$ D. Chae and O. Y. Imanuvilov, "Non-topological multivortex solutions to the self-dual Maxwell-Chern-Simons-Higgs systems," J. Funct. Anal. 196(1), 87-118 (2002).

${ }^{5}$ S.-H. Choi, S.-Y. Ha, and H. Lee, "Dispersion estimates for the two-dimensional Vlasov-Yukawa system with small data," J. Differ. Equ. 250, 515-550 (2011).

${ }^{6}$ S. Deser, R. Jackiw, and S. Templeton, "Three-dimensional massive gauge theories," Phys. Rev. Lett. 48, 975-978 (1982).

${ }^{7}$ G. Dunne, Self-dual Chern-Simons Theories (Springer, 1995).

${ }^{8}$ R. Glassey, The Cauchy Problem in Kinetic Theory (Society for Industrial and Applied Mathematics (SIAM), Philadelphia, PA, 1996).

${ }^{9}$ R. Glassey and W. Strauss, "Singularity formation in a collisionless plasma could occur only at high velocities," Arch. Rational Mech. Anal. 92(1), 59-90 (1986).

${ }^{10}$ R. Glassey and J. Schaeffer, "On the 'one and one-half dimensional' relativistic Vlasov-Maxwell system," Math. Methods Appl. Sci. 13(2), 169-179 (1990).

${ }^{11}$ R. Glassey and J. Schaeffer, "The 'two and one-half dimensional' relativistic Vlasov-Maxwell system," Commun. Math. Phys. 185, 257-284 (1997).

${ }^{12}$ R. Glassey and J. Schaeffer, "The relativistic Vlasov-Maxwell system in two space dimensions: Part I," Arch. Rational Mech. Anal. 141, 331-354 (1998).

${ }^{13}$ R. Glassey and J. Schaeffer, "The relativistic Vlasov-Maxwell system in two space dimensions: Part II," Arch. Rational Mech. Anal. 141, 355-374 (1998).

${ }^{14}$ J. Han and H. Huh, "Self-dual vortices in a Maxwell-Chern-Simons model with non-minimal coupling," Lett. Math. Phys. 82(1), 9-24 (2007).

${ }^{15}$ S.-Y. Ha, T. Ha, C.-O. Hwang, and H. Lee, "Nonlinear instability of the one-dimensional Vlasov-Yukawa system," J. Math. Phys. 52(3), 033301 (2011).

${ }^{16}$ S.-Y. Ha and H. Lee, "Global well posedness of the relativistic Vlasov-Yukawa system with small data," J. Math. Phys. 48(12), 123508 (2007).

${ }^{17}$ S.-Y. Ha and H. Lee, "Global existence of classical solutions to the damped Vlasov-Klein-Gordon equations with small data," J. Math. Phys. 50(5), 053302 (2009).

${ }^{18}$ J. Han and K. Song, "Existence and asymptotics of topological solutions in the self-dual Maxwell-Chern-Simons O(3) sigma model," J. Differ. Equ. 250(1), 204-222 (2011).

${ }^{19}$ M. Kunzinger, G. Rein, R. Steinbauer, and G. Teschl, "Global weak solutions of the relativistic Vlasov-Klein-Gordon system," Commun. Math. Phys. 238, 367-378 (2003). 
${ }^{20}$ M. Kunzinger, G. Rein, R. Steinbauer, and G. Teschl, "On classical solutions of the relativistic Vlasov-Klein-Gordon system,” Electron. J. Differential Equations 2005(01), 1-17 (2005).

${ }^{21} \mathrm{H}$. Lee, "The classical limit of the relativistic Vlasov-Maxwell system in two space dimensions," Math. Methods Appl. Sci. 27, 249-287 (2004).

${ }^{22}$ A. M. Polyakov, "Fermi-Bose transmutations induced by gauge fields," Mod. Phys. Lett. A 3(3), 325-328 (1988).

${ }^{23}$ R. E. Prange and S. M. Girvin, The Quantum Hall Effect (Springer, Berlin, 1987).

${ }^{24}$ G. N. Watson, A Treatise on the Theory of Bessel Functions (Cambridge University Press, London, 2008).

${ }^{25}$ E. Witten, "Quantum field theory and the Jones polynomial," Commun. Math. Phys. 121(3), 351-399 (1989).

${ }^{26}$ S. C. Zhang, T. H. Hansson, and S. Kivelson, "Effective-field-theory model for the fractional quantum hall effect," Phys. Rev. Lett. 62, 82-85 (1989). 